\title{
OPEN Comparative genome-wide analysis of WRKY, MADS-box and MYB transcription factor families in Arabidopsis and rice
}

\author{
Muhammad-Redha Abdullah-Zawawi ${ }^{1}$, Nur-Farhana Ahmad-Nizammuddin ${ }^{2}$, \\ Nisha Govender ${ }^{1 \bowtie}$, Sarahani Harun ${ }^{1}$, Norfarhan Mohd-Assaad ${ }^{2}$ \& \\ Zeti-Azura Mohamed-Hussein ${ }^{1,2}$
}

Transcription factors (TFs) form the major class of regulatory genes and play key roles in multiple plant stress responses. In most eukaryotic plants, transcription factor (TF) families (WRKY, MADSbox and MYB) activate unique cellular-level abiotic and biotic stress-responsive strategies, which are considered as key determinants for defense and developmental processes. Arabidopsis and rice are two important representative model systems for dicot and monocot plants, respectively. A comprehensive comparative study on 101 OsWRKY, 34 OsMADS box and 122 OsMYB genes (rice genome) and, 71 AtWRKY, 66 AtMADS box and 144 AtMYB genes (Arabidopsis genome) showed various relationships among TFs across species. The phylogenetic analysis clustered WRKY, MADSbox and MYB TF family members into 10, 7 and 14 clades, respectively. All clades in WRKY and MYB TF families and almost half of the total number of clades in the MADS-box TF family are shared between both species. Chromosomal and gene structure analysis showed that the Arabidopsis-rice orthologous TF gene pairs were unevenly localized within their chromosomes whilst the distribution of exon-intron gene structure and motif conservation indicated plausible functional similarity in both species. The abiotic and biotic stress-responsive cis-regulatory element type and distribution patterns in the promoter regions of Arabidopsis and rice WRKY, MADS-box and MYB orthologous gene pairs provide better knowledge on their role as conserved regulators in both species. Co-expression network analysis showed the correlation between WRKY, MADs-box and MYB genes in each independent rice and Arabidopsis network indicating their role in stress responsiveness and developmental processes.

Transcription factors (TFs) are characterized as proteins with at least one domain that corresponds to a specificDNA binding site and control the transcriptional regulatory schemes in plant cells. TFs regulate the spatiotemporal expression of target genes involved in plant growth and development, and response systems to the terrestrial environment. TF mediated responses are established upon intrinsic and external signals in controlling and coordinating the activation or repression of functional gene expression ${ }^{1-4}$. TFs have a unique DNA binding site, known as the cis-regulatory element (CREs) in the promoter region of a gene for independent regulation, induction and/or cross-regulatory activation such as epigenetics and signalling process. TFs are categorized according to the conserved motifs in DNA-binding domains (DBDs) such as NAC, SBP, MADS-box, WRKY, B3 among others. In plants, the distribution of TF families is assumed plant species-specific. Currently, 58 different TF families are deposited in the PlantTFDB database and they have been exclusively characterized in model plants $^{3}$. Amongst these TF families, WRKY, MADS-box and MYB are the most important transcriptional regulators that are widely distributed in the plant kingdom and actively involved in plant development and, biotic and abiotic stress responses ${ }^{4}$.

The WRKY, the seventh-largest family of TFs is involved in the developmental processes and defense responses such as seed germination, pollen development, hormonal regulation, biosynthesis of secondary metabolites ${ }^{5}$. WRKY TF family is characterized by a WRKY signature domain that contains WD containing amino acid residues positioned at the $\mathrm{N}$-terminus and a zinc-finger domain at the $\mathrm{C}$-terminus of the sequence. It consists

${ }^{1}$ Institute of Systems Biology (INBIOSIS), Universiti Kebangsaan Malaysia, 43600 UKM, Bangi, Selangor, Malaysia. ${ }^{2}$ Department of Applied Physics, Faculty of Science and Technology, Universiti Kebangsaan Malaysia, 43600 UKM, Bangi, Selangor, Malaysia. ${ }^{\circledR}$ email: nishag@ukm.edu.my 
of approximately 60-70 amino acid residues with WRKYGQK /WRKYGKK motif for DNA-binding promoter element or W-Box (TTGACC/T) recognition ${ }^{6,7}$. In the MYB family, TFs are involved in plant development and defense responses including cell cycle, cell morphogenesis, central circadian oscillator and regulation of stress signalling ${ }^{8,9}$. The MYB domain contains three irregular repeats that form a helix-turn-helix (HTH) structure of about 53 amino acids ${ }^{10}$. In MYB proteins, the R1, R2, R3 (conventional) and R4 groups (numbered according to the number of the adjacent repeats) of MYB-domain repeats stabilize the DNA-binding structure ${ }^{11}$. The TFs with MCM1/AGAMOUS/DEFICIENS/SRF (MADS)-box regulate the developmental processes such as seed germination, vegetative growth, the transition from vegetative to reproductive growth, floral development and senescence and regulating the abiotic and biotic stress tolerance. They contain a conserved MADS domain consists of 60-amino acid long at the N-terminal and recognizes the CArG-box DNA motif (CC[A/ $\left.]_{6} \mathrm{GG}\right)$ in the target genes. Generally, they are classified into two lineages namely, type I and type II. Type 1 contains MADS domain and an extended highly variable carboxy-terminal domain whilst type II contains four conserved domains known as the MIKC that consists of M-domain, Intervening-domain, Keratin-like domain and the carboxy-terminal domain ${ }^{12}$.

Rice and Arabidopsis are important non-halophytes model plants for monocot and dicot crops, respectively. They are short-rotation plants with high sensitivity to stressors; oxidative, osmotic and ion/salt stress ${ }^{13,14}$. The first rice genome was published in 2006 and has become an excellent model system for the economically important related monocotyledons crops such as maize, wheat, sorghum and barley. On the other hand, the dicotyledonous $A$. thaliana was the first model plant with a completed genome sequence published in the year 2000 (http:// www.arabidopsis.org $)^{13}$. It has been actively used by the plant research community in revolutionizing genetics and breeding studies ${ }^{14}$. More than $5 \%$ of the Arabidopsis genes encode for TFs and only about $7 \%$ of them have been functionally and genetically characterized. The genome size of Arabidopsis is approximately 135 megabase pairs, about one-fourth of the size of the rice genome and contains up to 30000 genes. Currently, there are 2296 and 2408 genes encoding TFs in Arabidopsis and rice, respectively ${ }^{15}$.

The Arabidopsis and rice WRKY, MADS-box and MYB TF families are reported to show diverse functional roles. In rice, the OsMYB-R1 gene regulates multiple stress tolerance ${ }^{16}$, RADIALS-LIKE3 (OsRL3) promotes dark-induced leaf senescence and reduce susceptibility to salt stress ${ }^{17}$, OsWRKY74 and OsWRKY28 regulate the phosphate homeostasis ${ }^{18,19}$ and OsMADS27 regulates root development under a salt-tolerant condition ${ }^{20}$. In Arabidopsis, AGL21, the MADS-box TF acts as environmental surveillance during seed germination. There are 109 and 74 WRKY families in rice and Arabidopsis, respectively ${ }^{21}$. The MYB TF family with up to180 members is the largest TF family in Arabidopsis and rice ${ }^{9}$. The MADS-box TF family contains more than 100 members and are generally involved in almost every developmental process of a higher plant ${ }^{22}$.

TFs are an important component in complex regulatory networks established by plants during their response to stressors ${ }^{19-21}$. They either enhance or suppress the expression of genes that are directly associated with target resistance genes. In this study, the WRKY, MADS-box and MYB TF families from rice and Arabidopsis were identified and collated for a comprehensive in silico genome-wide analysis in the search for conserved functional roles between different TF families and species. The phylogenetic relationship of the exon-intron arrangement, conserved motif analysis, and promoter analysis of stress-responsive cis-regulatory elements present in the orthologous gene pairs (Arabidopsis and rice) of three WRKY, MADS-box and MYB TF families are investigated to provide useful insights on the conserved regulatory modules of TFs with potential manipulation for plant biotechnology and breeding programmes.

\section{Materials and methods}

Data resources. Genes of Arabidopsis thaliana and Oryza sativa WRKY, MADS-box and MYB encoding transcription factors (TFs) were retrieved from Plant Transcription Factor Database v5.0 (PlantTFDB 5.0; http:// planttfdb.cbi.pku.edu.cn $)^{15}$. The corresponding protein-coding sequences were obtained from Phytozome 12.1 (https://phytozome.jgi.doe.gov/pz/portal.html) ${ }^{23}$.

Multiple sequence alignment and phylogenetic analysis. The multiple sequence alignment (MSA) was conducted using ClustalW v2.1 software with the following parameters set: open penalty of 10 gaps and gap extension at 0.1 to $0.2^{24}$ followed by the phylogenetic tree construction using MEGA v7.2 software with the Neighbor-Joining (NJ) method with 1000 bootstrap replicates ${ }^{25,26}$. The phylogenetic tree was visualized and annotated using FigTree software v1.4.4 (http://tree.bio.ed.ac.uk/software/figtree/) ${ }^{27}$.

Chromosomal location analysis. The chromosomal location analysis of the WRKY, MADS-box and MYB TF gene families were performed using TAIR Chromosome Map Tool (https://www.arabidopsis.org/jsp/ ChromosomeMap/tool.jsp) ${ }^{28}$ for Arabidopsis and Oryzabase Chromosome Map Tool (http://viewer.shigen.info/ oryzavw/maptool/MapTool.do) for rice ${ }^{29}$. Genes separated by less than five gene loci at $100 \mathrm{~kb}$ distance were considered as tandem duplicates ${ }^{30}$.

Exon-intron arrangement and motifs search distributions. The exon-intron structural features of WRKY, MADS-box and MYB TF genes were visualized using Gene Structure Display Server 2.0 (http://gsds. cbi.pku.edu.cn/ $/)^{31}$. The conserved motifs of the target sequences were identified by Multiple Expectation Maximization for Motif Elicitation (MEME) Suite Software (http://meme-suite.org/) using the following parameters: maximum number motifs is set at 20 and allow zero or one occurrence per sequence (zoops) mode ${ }^{32}$. Pfam online tool (https://pfam.xfam.org) was employed for conserved motif annotation ${ }^{33}$. 
Prediction of cis-regulatory element on promoter regions. Promoter region and the cis-regulatory elements (CREs) of the WRKY, MADS-box and MYB target sequences were examined using a web-based tool, the PLANTCARE (http://bioinformatics.psb.ugent.be/webtools/plantcare/html) ${ }^{34}$ followed by the visualization of CREs using Illustrator for Biological Sequences (IBS) software (http://ibs.biocuckoo.org) $)^{35}$.

In silico co-expression analysis and functional similarity between orthologous gene pair. Gene identifier of orthologous pair for WRKY, MADS-box and MYB target sequences was searched against PLANT co-expression database (PLANEX, http://planex.plantbioinformatics.org) ${ }^{36}$. The co-expression data were retrieved, and the networks were visualized using Cytoscape v3.7.0 software ${ }^{37}$. Functional similarity of the coexpression network was measured using kappa value from PLANEX database ${ }^{36}$ that represents the distance of co-expression data between rice and Arabidopsis.

\section{Results \\ Phylogenetic analysis of WRKY, MADS-box and MYB genes in Arabidopsis and rice. 101 OsWRKY, 34 OsMADS box and 122 OsMYB sequences were identified in rice and 72 AtWRKY, 66 AtMADS box and 144 AtMYB sequences were identified in Arabidopsis after the repetitive and redundant gene sequences were removed. A phylogenetic tree for the WRKY transcription factor (TF) family was built from 173 collated Arabidopsis and rice WRKY genes. 101 OsWRKY and 72 AtWRKY genes are distributed in all clades except clade 5 where only one Arabidopsis gene (AtWRKY) is present among 22 rice genes $(O s W R K Y)$ whilst Clade 10 contains WRKY genes from rice only. The highest gene number $(\mathrm{GN})$ is observed in clade $8(\mathrm{GN}=37)$, followed by clade $6(\mathrm{GN}=26)$, clade $7(\mathrm{GN}=24)$ and clade $5(\mathrm{GN}=23)$. Clade 9 is the smallest with a $\mathrm{GN}=3$ (Fig. 1). A phylogenetic tree of the MADS-box TF family constructed from 66 AtMADS-box and 34 OsMADS-box genes shows consistent distribution among 14 clades. Clade 1 and clade 7 are the biggest clusters with a similar size $(\mathrm{GN}=25)$, followed by clade $6(\mathrm{GN}=20)$, clade $2(\mathrm{GN}=15)$, clade $(\mathrm{GN}=9)$ and clade $5(\mathrm{GN}=5)$. Clade 4 is the smallest with $\mathrm{GN}=2$. Clade 3 and clade 6 contain gene members from AtMADS-box only while clade 4 and clade 5 are unique to OsMADS-box members (Fig. 2). A phylogenetic tree of the MYB TF family shows 14 clades, with fairly even Arabidopsis and rice genes representation. Clade 1 is the biggest cluster $(\mathrm{GN}=54)$, followed by clade $10(\mathrm{GN}=29)$, clade $7(\mathrm{GN}=27)$, clade $4(\mathrm{GN}=25)$ and clade $7(\mathrm{GN}=6)(\mathrm{Fig} .3)$. In each $\mathrm{TF}$ family phylogenetic tree, the orthologous gene pairs identified by red circles were selected for subsequent analysis. A total of 22 orthologous gene pairs are obtained as following: WRKY;10, MADS-box; 1 and MYB; 11 (Figs. 1, 2, 3).}

Distribution of the WRKY, MADS-box and MYB orthologous genes in Arabidopsis and Oryza sativa chromosomes. The in silico mapping of WRKY, MADS-box and MYB orthologous gene pairs showed an uneven distribution in Oryza sativa (Os) and Arabidopsis thaliana (At) chromosomes (Chr). In Arabidopsis, the orthologous genes were distributed randomly in AtChr1, AtChr2, AtChr3, AtChr4 and AtChr5. A total of five genes, one from MADS-box, two each from MYB and WRYK TF families were located on AtChr1. On AtChr2 and AtChr4, three WRKY and one MYB genes were located at various distances. All four genes located on AtChr3 are from the MYB family. The AtChr5 showed a random distribution of three MYB and two WRYK genes. In rice, the orthologous genes were present on almost every chromosome except OsChr6, OsChr9 and OsChr10. The OsChr1 contain the highest gene number (GN) at 7, followed by OsChr4 $(\mathrm{GN}=3)$ and $\mathrm{OsChr} 7(\mathrm{GN}=3)$, and $\mathrm{OsChr} 8, \mathrm{OsChr} 11$ and $\mathrm{OsChr} 12$ with $\mathrm{GN}=2$ each. The least number of genes were distributed in OsChr2, OsChr3 and OsChr5 (GN =1) (Fig. 4). Detailed distribution of WRKY, MADS-box and MYB orthologous genes on Arabidopsis and rice chromosomes are shown in Table 1. Separated by at least more than five gene loci, no tandem duplications were observed among the genes. The longest protein was encoded by AtWRKY1 (1789 aa) in Arabidopsis and OsMYB50 (72 aa) in rice. Likewise, the shortest protein was encoded by AtWRKY43 (109 aa) and OsWRKY58 (181 aa). More than half of the proteins encoded by AtMYB and $O s M Y B$ genes were acidic with a theoretical isoelectric point value of less than7 whilst two MADS-box proteins (AtAGL65 and OsMADS68) were acidic. A total of 8 OsWRKY proteins were acidic in comparison to 2 from AtWRKY. The average molecular weight (MW) of these proteins were $48.7 \mathrm{kDa}$ and $45.4 \mathrm{kDa}$ in Arabidopsis and rice, respectively. Detailed information on the sequence characteristics is given in Table 1.

Gene structure and conserved motif analysis: WRKY, MADS-box and MYB orthologous genes in Arabidopsis and rice. A total of 173 WRKY, 100 MADS-box and 266 MYB genes were identified with distinctive exon number (EN) and intron number (IN). Among the WRKY genes, EN ranged at 1-15. A total of 95 genes showed $\mathrm{EN}=3$ and 88 genes showed $\mathrm{IN}=3,22$ genes with $\mathrm{EN}=2$, and 22 genes with $\mathrm{EN}=2$ and $\mathrm{IN}=4$. The AT4G12020 gene showed the highest EN and IN with 15 and 14, respectively. Meanwhile, 63 MADS-box genes showed $\mathrm{EN}=1,16$ genes with $\mathrm{IN}=1$, and 13 genes with $\mathrm{EN}=2$, and eight genes with $\mathrm{IN}=2$. Among the MYB genes, 156 genes showed $\mathrm{EN}=3,154$ genes with $\mathrm{IN}=2,58$ genes with $\mathrm{EN}=2$, and 57 genes with $\mathrm{IN}=1$ (Supplementary File: Figs. 1, 2, 3). Generally, MADS-box $(\mathrm{EN}=1-11)$ and MYB $(\mathrm{EN}=1-13)$ genes showed a similar range of ENs. Comparatively, the rate of EN and IN difference in the WRKY and MYB TF families was higher than the MADS-box. The exon-intron structure of the ortholog and paralog pairs were further examined. Dissimilarities in the number of exons among the following orthologous gene pairs suggest either a protein gain or loss event in both species: (i) LOC_Os01g54600- AT1G29280, (ii) LOC_Os02g53100- AT1G68150, (iii) LOC_Os11g43740- AT1G18750, and iv) LOC_Os12g38400- AT2G37630. The rice LOC_Os01g54600, LOC_ Os02g53100, LOC_Os11g43740 and LOC_Os12g38400 genes were identified to gain one exon whilst their counterpart pairs, AT1G29280, AT1G68150, AT1G18750 and AT2G37630 showed a lost one exon (Fig. 5).

A total of 20 distinct conserved motifs were identified in Arabidopsis and rice orthologous genes comprised of 20 WRKY, two MADS-box, and 22 MYB proteins. Almost all orthologous genes, the same type of motifs were 


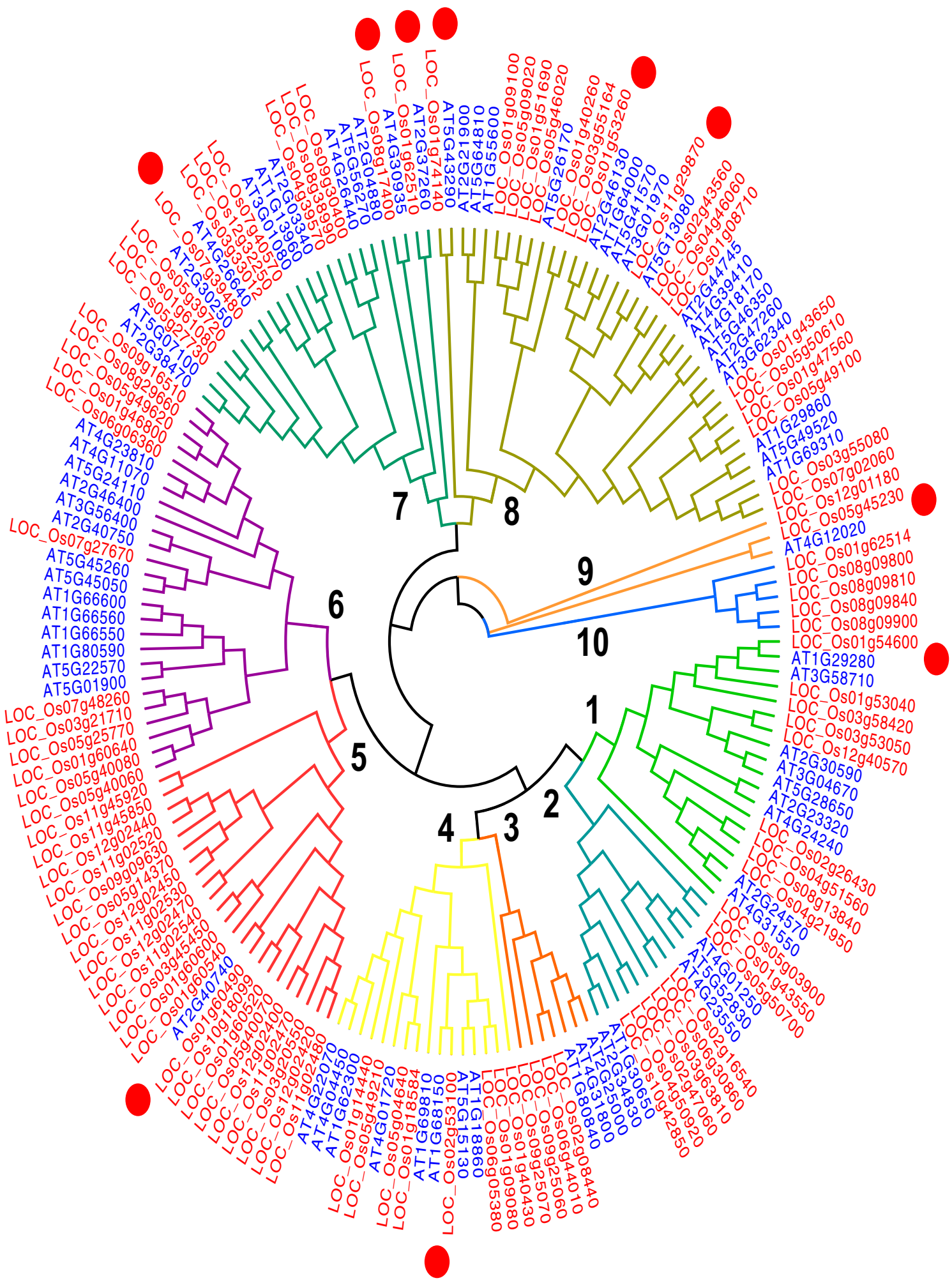

Figure 1. Phylogenetic tree of collated rice and Arabidopsis full-length WRKY protein sequences. Red dots represent the rice-Arabidopsis orthologous gene pairs. The tree is built using the neighbor-joining (NJ) method (MEGA7.0 software) and are divided into ten clades, numbered in bold.

present in each gene sequence with different distribution patterns. Evaluation by transcription factor family shows that genes in a common clade shared a closely similar pattern of motif distributions (Fig. 6). The WRKY TF family shows apparent motif similarity with the genes in clade1, 4, 5, 7 and 8 except clade 9. Each clade contains 


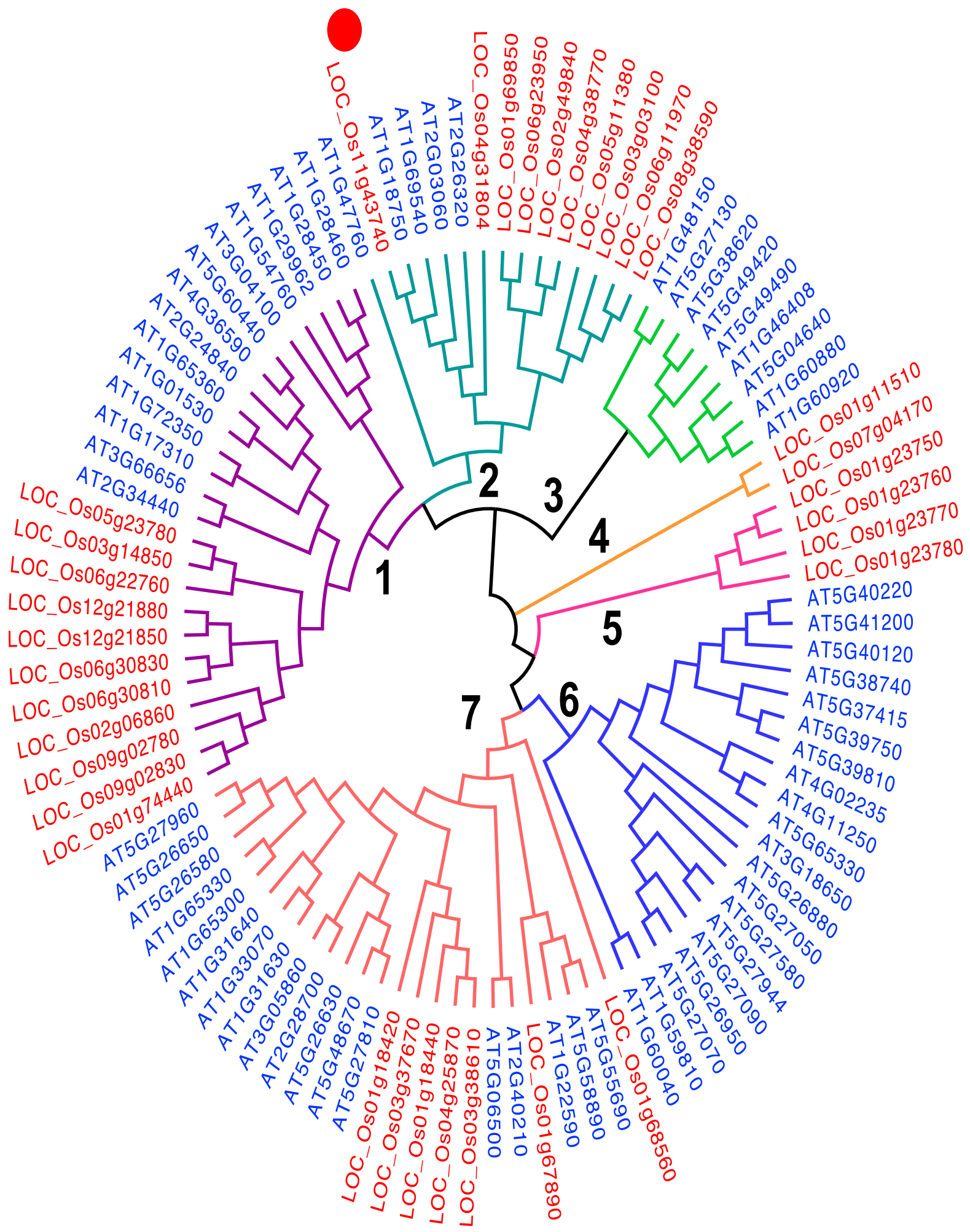

Figure 2. Phylogenetic tree of collated rice and Arabidopsis full-length MADS-box protein sequences. Red dots represent the rice-Arabidopsis orthologous gene pairs. The tree is built using the neighbor-joining (NJ) method (MEGA7.0 software) and are divided into seven clades, numbered in bold.

various number of motifs with unique distribution patterns. In the MYB TF family, clade 1-10 were similar with at least 3 identical conserved motifs. Clade 12 showed the highest number of motifs and clade 13 showed the least number. Motif 1 was present within the MYB TF family members whereas motif 2 was found in all clades except clades 12 and 13. The MADS-box TF family represented by a pair of orthologous genes contained 


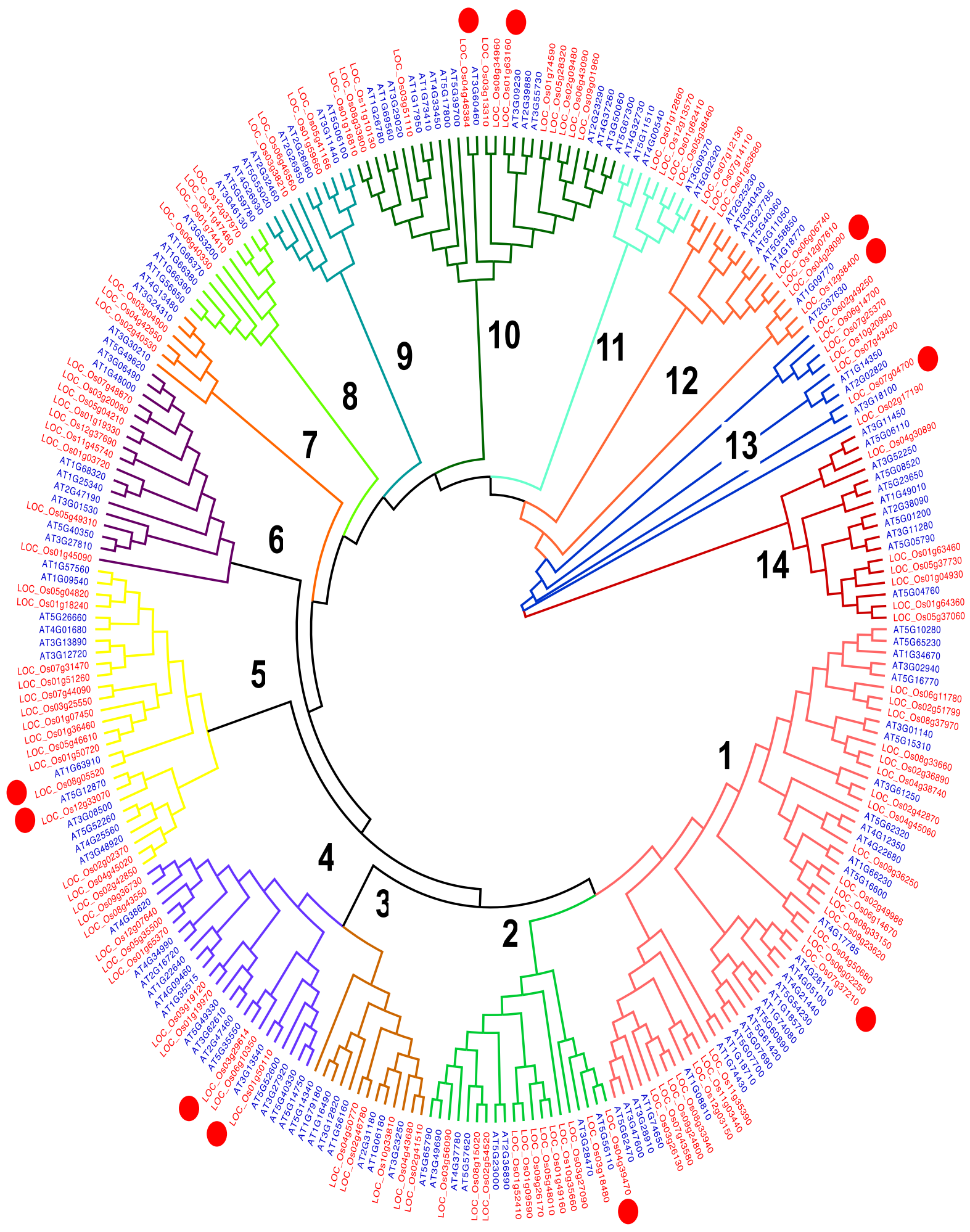

Figure 3. Phylogenetic tree of collated rice and Arabidopsis full-length MYB protein sequences. Red dots represent the rice-Arabidopsis orthologous gene pairs. The tree is built using the neighbor-joining (NJ) method (MEGA7.0 software) and are divided into 14 clades, numbered in bold.

20 different motifs distributed in a similar pattern. Detailed information on motif function annotation of the motifs identified in the WRKY, MYB and MADS-box TF family rice-Arabidopsis orthologous genes is presented in Supplementary File 2: Table 1. 
(A)

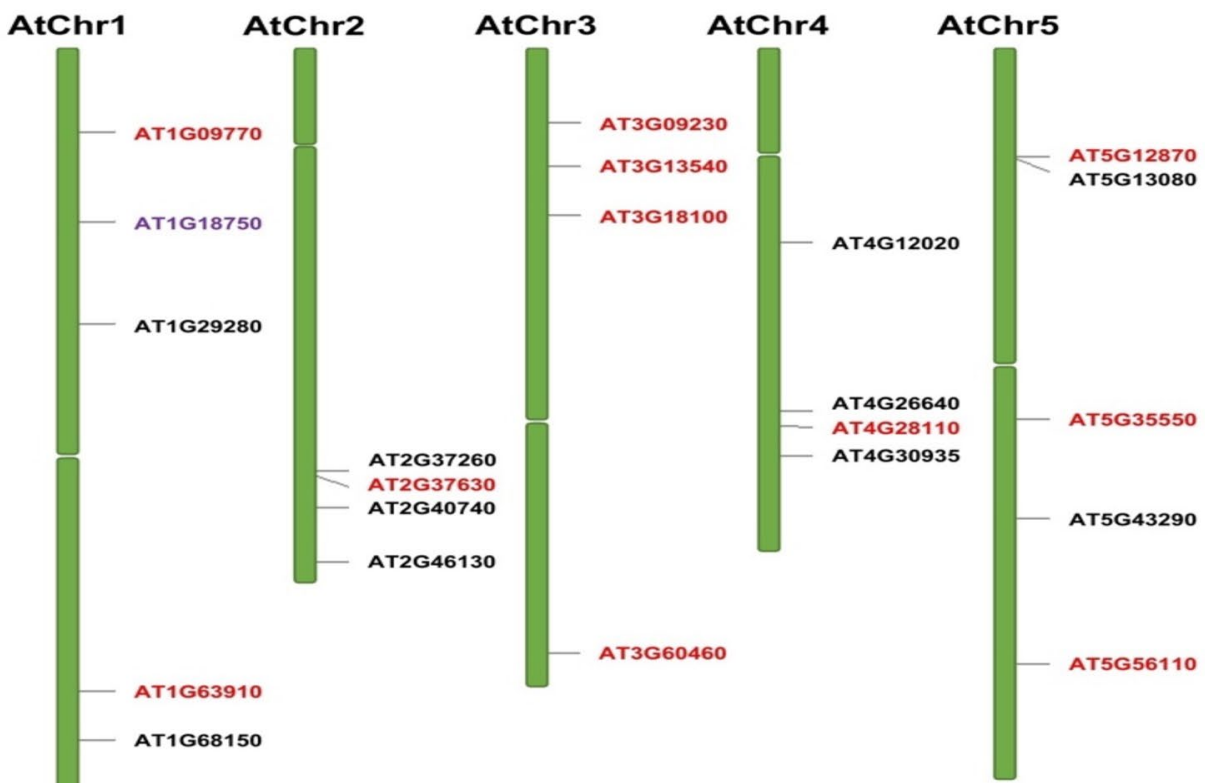

(B)

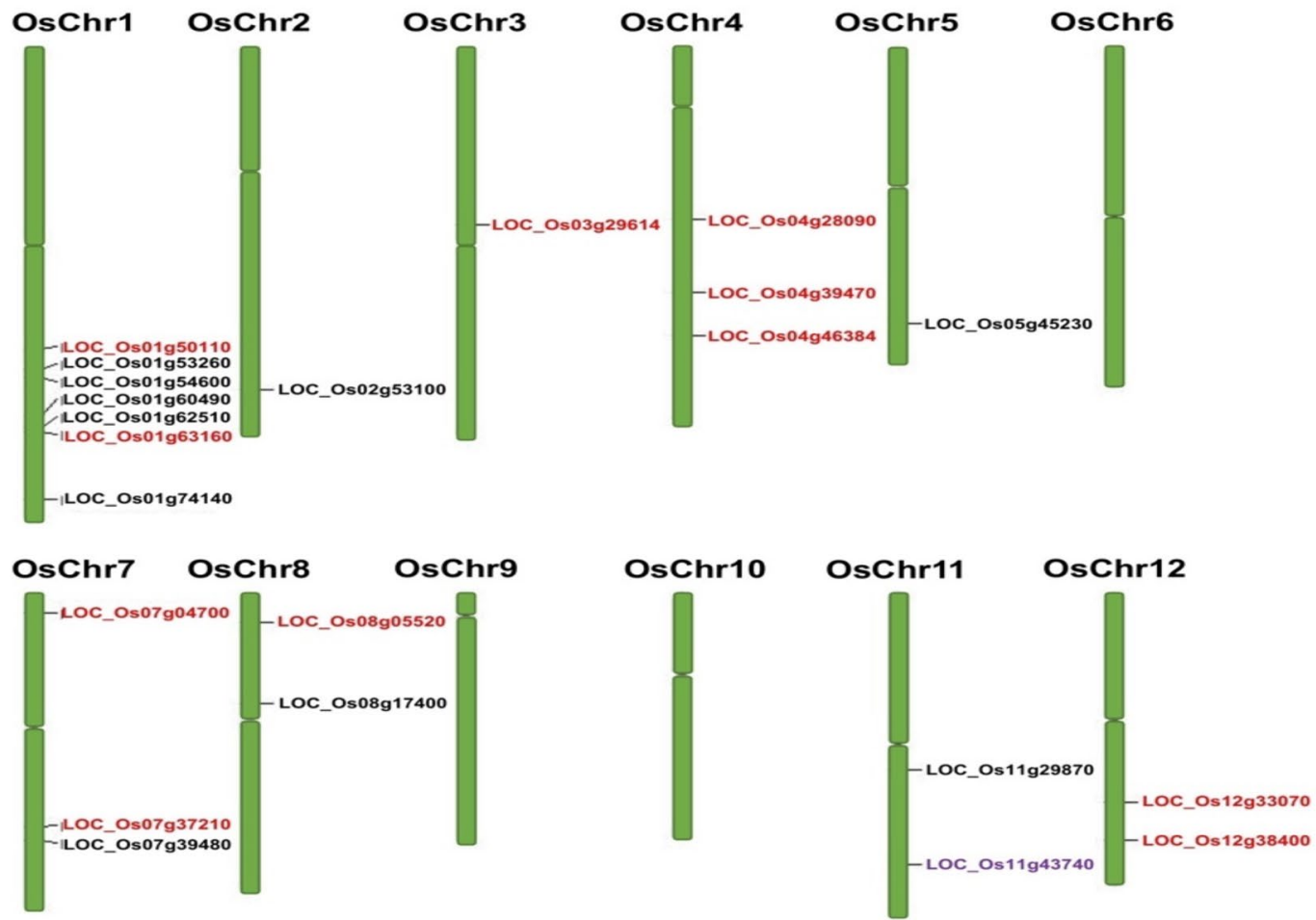

Figure 4. The chromosomal distribution of rice-Arabidopsis WRKY, MADS-box and MYB orthologous gene. (A) Distribution of gene loci on Arabidopsis chromosomes. (B) Distribution of gene loci on rice chromosomes. Different gene loci colours (naming) represents a gene transcription factor family: WRKY; black, MADS-box; purple and MYB; red.

Distribution of cis-regulatory elements (CREs) in putative promoter regions of Arabidopsis and rice orthologous WRKY, MADS-box and MYB genes. The orthologous Arabidopsis and rice genes (WRKY, MADS-box and MYB TF family) were screened for cis-regulatory elements (CREs) distribution within the sequence. The CREs were randomly distributed in positive and negative strands of the promoter region of the gene sequence. Comprehensive details of the CREs identified in Arabidopsis and rice WRKY, MADS-box and MYB orthologous genes are presented in Supplementary File 4. In rice, the most abundant CREs were encoding for jasmonate-responsive signalling (CGTCA-motif and TGACG-motif), light-responsive (Sp1 and G-box) 


\begin{tabular}{|c|c|c|c|c|c|c|c|c|}
\hline \multirow[b]{2}{*}{ Gene identifier } & \multirow[b]{2}{*}{ Name $^{*}$} & \multirow[b]{2}{*}{ Chr } & \multirow[b]{2}{*}{ Location } & \multirow[b]{2}{*}{ ORF length (bp) } & \multicolumn{3}{|l|}{ Protein } & \multirow[b]{2}{*}{ Exon number } \\
\hline & & & & & Length & PI & $\begin{array}{l}\text { Molecular } \\
\text { weight (Da) }\end{array}$ & \\
\hline AT1G18750 & AtAGL65 ${ }^{1}$ & 1 & $\begin{array}{l}6,466,761- \\
6,469,984\end{array}$ & 1170 & 389 & 6.504 & $44,877.5$ & 10 \\
\hline AT4G28110 & 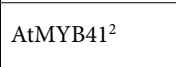 & 4 & $\begin{array}{l}13,968,029- \\
13,969,384\end{array}$ & 849 & 282 & 5.903 & $31,651.6$ & 3 \\
\hline AT5G56110 & AtMYB $80^{3}$ & 5 & $\begin{array}{l}22,719,191- \\
22,720,664\end{array}$ & 963 & 320 & 7.322 & $35,983.4$ & 3 \\
\hline AT3G13540 & AtMYB5 ${ }^{4}$ & 3 & \begin{tabular}{|l|}
$4,420,173-$ \\
$4,421,701$
\end{tabular} & 750 & 249 & 8.285 & $27,793.5$ & 2 \\
\hline AT5G35550 & AtMYB $123^{5}$ & 5 & $\begin{array}{l}13,726,743- \\
13,727,860\end{array}$ & 777 & 258 & 8.903 & $29,611.4$ & 3 \\
\hline AT5G12870 & AtMYB46 $6^{6}$ & 5 & \begin{tabular}{|l|}
$4,062,724-$ \\
$4,064,992$
\end{tabular} & 843 & 280 & 6.037 & $31,541.3$ & 2 \\
\hline AT1G63910 & AtMYB $103^{7}$ & 1 & $\begin{array}{l}23,719,783- \\
23,721,774\end{array}$ & 1113 & 370 & 5.681 & $42,262.6$ & 3 \\
\hline AT3G60460 & AtMYB $125^{8}$ & 3 & $\begin{array}{l}22,342,429- \\
22,343,491\end{array}$ & 894 & 297 & 6.075 & $33,649.6$ & 3 \\
\hline AT3G09230 & AtMYB $1^{9}$ & 3 & \begin{tabular}{|l|}
$2,833,398-$ \\
$2,835,338$
\end{tabular} & 1182 & 393 & 5.217 & $42,811.4$ & 2 \\
\hline AT1G09770 & AtMYBCDC $5^{10}$ & 1 & $\begin{array}{l}3,161,841- \\
3,165,360\end{array}$ & 2535 & 844 & 6.731 & $95,766.6$ & 4 \\
\hline AT2G37630 & AtMYB91 $1^{11}$ & 2 & $\begin{array}{l}15,781,615- \\
15,783,433\end{array}$ & 1104 & 367 & 9.555 & $42,243.1$ & 1 \\
\hline AT3G18100 & AtMYB4R $1^{12}$ & 3 & \begin{tabular}{|l|}
$6,200,524-$ \\
$6,204,644$
\end{tabular} & 2544 & 847 & 5.580 & $96,084.4$ & 7 \\
\hline AT1G29280 & AtWRKY $65^{13}$ & 1 & \begin{tabular}{|l|}
$10,236,367-$ \\
$10,237,467$
\end{tabular} & 780 & 259 & 5.469 & $29,054.4$ & 2 \\
\hline AT1G68150 & AtWRKY9 ${ }^{14}$ & 1 & $\begin{array}{l}25,543,969- \\
25,545,717\end{array}$ & 1125 & 374 & 7.816 & $42,743.0$ & 5 \\
\hline AT2G40740 & AtWRKY55 ${ }^{15}$ & 2 & \begin{tabular}{|l|}
$16,997,177-$ \\
$16,999,277$ \\
\end{tabular} & 879 & 292 & 8.049 & $32,488.8$ & 3 \\
\hline AT4G26640 & AtWRKY20 ${ }^{16}$ & 4 & \begin{tabular}{|l|}
$13,437,071-$ \\
$13,440,835$
\end{tabular} & 1458 & 485 & 7.102 & $53,601.5$ & 5 \\
\hline AT4G30935 & AtWRKY $32^{17}$ & 4 & \begin{tabular}{|l|}
$15,051,814-$ \\
$15,054,042$
\end{tabular} & 1401 & 466 & 5.895 & $51,480.4$ & 5 \\
\hline AT2G37260 & AtWRKY $44^{18}$ & 2 & $\begin{array}{l}15,644,840- \\
15,647,065\end{array}$ & 1290 & 429 & 9.399 & $47,141.2$ & 4 \\
\hline AT5G43290 & AtWRKY $49^{19}$ & 5 & $\begin{array}{l}17,371,838- \\
17,373,201 \\
\end{array}$ & 825 & 274 & 7.924 & $31,580.6$ & 3 \\
\hline AT2G46130 & AtWRKY $43^{20}$ & 2 & $\begin{array}{l}18,957,226- \\
18,957,911 \\
\end{array}$ & 330 & 109 & 9.992 & $12,951.8$ & 2 \\
\hline AT5G13080 & AtWRKY75²1 & 5 & $\begin{array}{l}4,149,740- \\
4,151,150\end{array}$ & 438 & 145 & 9.593 & $16,801.8$ & 2 \\
\hline AT4G12020 & AtWRKY1922 & 4 & $\begin{array}{l}7,201,656- \\
7,209,648\end{array}$ & 5397 & 1798 & 7.019 & $199,996.0$ & 15 \\
\hline $\begin{array}{l}\text { LOC }_{-} \\
\text {Os11g43740 }\end{array}$ & OsMADS68 ${ }^{1}$ & 11 & $\begin{array}{l}26,414,394- \\
26,418,442\end{array}$ & 1179 & 392 & 6.829 & $43,366.9$ & 11 \\
\hline $\begin{array}{l}\text { LOC }_{-} \\
\text {Os07g37210 }\end{array}$ & OsMYB102 2 & 7 & \begin{tabular}{|l|}
$22,293,735-$ \\
$22,295,309$
\end{tabular} & 1107 & 368 & 7.092 & $39,929.0$ & 3 \\
\hline $\begin{array}{l}\text { LOC }_{-} \\
\text {Os04g39470 }\end{array}$ & OsMYB $80^{3}$ & 4 & $\begin{array}{l}23,510,412- \\
23,512,029\end{array}$ & 1119 & 372 & 6.146 & $39,699.2$ & 3 \\
\hline $\begin{array}{l}\text { LOC_ } \\
\text { Os01g50110 }\end{array}$ & OsMYB13 ${ }^{4}$ & 1 & $\begin{array}{l}28,796,516- \\
28,797,732\end{array}$ & 828 & 275 & 6.107 & $29,793.3$ & 2 \\
\hline $\begin{array}{l}\text { LOC_ } \\
\text { Os03g29614 }\end{array}$ & OsMYB $46^{5}$ & 3 & $\begin{array}{l}16,879,442- \\
16,883,640\end{array}$ & 966 & 321 & 6.624 & 34,049 & 3 \\
\hline $\begin{array}{l}\text { LOC_ } \\
\text { Os12g33070 }\end{array}$ & OsMYB $122^{6}$ & 12 & $\begin{array}{l}19,991,426- \\
19,994,401\end{array}$ & 1230 & 409 & 6.824 & $43,722.4$ & 2 \\
\hline $\begin{array}{l}\text { LOC_ } \\
\text { Os08g05520 }\end{array}$ & OsMYB937 & 8 & $\begin{array}{l}2,948,522- \\
2,951,372\end{array}$ & 1080 & 359 & 6.624 & $39,954.7$ & 3 \\
\hline $\begin{array}{l}\text { LOC }_{-} \\
\text {Os04g46384 }\end{array}$ & OsMYB58 ${ }^{8}$ & 4 & $\begin{array}{l}27,503,041- \\
27,504,784\end{array}$ & 1032 & 343 & 7.919 & $37,110.9$ & 3 \\
\hline $\begin{array}{l}\text { LOC_ } \\
\text { Os01g63160 }\end{array}$ & OsMYB $19^{9}$ & 1 & $\begin{array}{l}36,606,535- \\
36,608,135\end{array}$ & 1242 & 413 & 6.697 & $44,329.6$ & 2 \\
\hline $\begin{array}{l}\text { LOC }_{-} \\
\text {Os04g28090 }\end{array}$ & OsMYB50 $0^{10}$ & 4 & $\begin{array}{l}16,579,869- \\
16,587,180\end{array}$ & 2919 & 972 & 4.878 & 109,684 & 4 \\
\hline $\begin{array}{l}\text { LOC_ } \\
\text { Os12g38400 }\end{array}$ & OsMYB125 $5^{11}$ & 12 & $\begin{array}{l}23,554,928- \\
23,560,551\end{array}$ & 1029 & 342 & 10.28 & $39,041.6$ & 2 \\
\hline
\end{tabular}




\begin{tabular}{|c|c|c|c|c|c|c|c|c|}
\hline \multirow[b]{2}{*}{ Gene identifier } & \multirow[b]{2}{*}{ Name $^{*}$} & \multirow[b]{2}{*}{ Chr } & \multirow[b]{2}{*}{ Location } & \multirow[b]{2}{*}{ ORF length (bp) } & \multicolumn{3}{|l|}{ Protein } & \multirow[b]{2}{*}{ Exon number } \\
\hline & & & & & Length & PI & $\begin{array}{l}\text { Molecular } \\
\text { weight }(\mathrm{Da})\end{array}$ & \\
\hline $\begin{array}{l}\text { LOC }_{-} \\
\text {Os07g04700 }\end{array}$ & OsMYB $87^{12}$ & 7 & $\begin{array}{l}2,084,106- \\
2,091,653\end{array}$ & 2907 & 968 & 8.639 & $106,868.0$ & 13 \\
\hline $\begin{array}{l}\text { LOC_ }_{-} \\
\text {Os01g54600 }\end{array}$ & OsWRKY $13^{13}$ & 1 & $\begin{array}{l}31,409,004- \\
31,410,978\end{array}$ & 951 & 316 & 4.601 & $34,294.6$ & 3 \\
\hline $\begin{array}{l}\mathrm{LOC}_{-} \\
\text {Os02g53100 }\end{array}$ & OsWRKY $32^{14}$ & 2 & $\begin{array}{l}32,489,017- \\
32,495,070\end{array}$ & 1815 & 604 & 4.800 & $62,940.3$ & 6 \\
\hline $\begin{array}{l}\text { LOC_ }_{-} \\
\text {Os01g60490 }\end{array}$ & OsWRKY $22^{15}$ & 1 & $\begin{array}{l}34,981,468- \\
34,985,447\end{array}$ & 798 & 265 & 7.110 & $29,807.4$ & 3 \\
\hline $\begin{array}{l}\text { LOC_ }_{-} \\
\text {Os07g39480 }\end{array}$ & OsWRKY $87^{16}$ & 7 & $\begin{array}{l}23,654,076- \\
23,659,625\end{array}$ & 1857 & 618 & 6.332 & $66,163.6$ & 6 \\
\hline $\begin{array}{l}\text { LOC_ } \\
\text { Os08g17400 }\end{array}$ & OsWRKY89 $9^{17}$ & 8 & $\begin{array}{l}10,633,195- \\
10,639,603\end{array}$ & 1653 & 550 & 6.707 & $59,781.9$ & 4 \\
\hline $\begin{array}{l}\text { LOC_ }_{-} \\
\text {Os01g62510 }\end{array}$ & OsWRKY119 18 & 1 & $\begin{array}{l}36,188,702- \\
36,191,681\end{array}$ & 612 & 203 & 5.042 & $21,483.5$ & 2 \\
\hline $\begin{array}{l}\text { LOC_ }_{-} \\
\text {Os01g74140 }\end{array}$ & OsWRKY $17^{19}$ & 1 & $\begin{array}{l}42,946,753- \\
42,948,750\end{array}$ & 1233 & 410 & 4.685 & $45,109.9$ & 3 \\
\hline $\begin{array}{l}\text { LOC }_{-} \\
\text {Os01g53260 }\end{array}$ & OsWRKY $23^{20}$ & 1 & $\begin{array}{l}30,604,295- \\
30,608,077\end{array}$ & 765 & 254 & 6.903 & $27,796.2$ & 2 \\
\hline $\begin{array}{l}\text { LOC }_{-} \\
\text {Os11g29870 }\end{array}$ & OsWRKY72 ${ }^{21}$ & 11 & $\begin{array}{l}17,352,085- \\
17,355,820\end{array}$ & 729 & 242 & 9.335 & $25,857.2$ & 2 \\
\hline $\begin{array}{l}\text { LOC_ }_{-} \\
\text {Os05g45230 }\end{array}$ & OsWRKY58 $8^{22}$ & 5 & $\begin{array}{l}26,256,951- \\
26,257,809\end{array}$ & 546 & 181 & 4.631 & $18,481.3$ & 2 \\
\hline
\end{tabular}

Table 1. Orthologous WRKY, MADS-box and MYB gene-pairs in Arabidopsis and rice. Each gene is described according to chromosome loci, open reading frame (ORF) length, properties of the encoding protein and exon number. ${ }^{\star}$ Similar superscript numbers in the name column represents orthologous gene pairs.

and plant development (GC-motif) whereas, in Arabidopsis, biotic and abiotic stress-responsive elements such as MYB, ABRE, STRE, As- 1 and MYC are distributed within the TF family genes. The stress-responsive CRE, ABRE is present in both species, whereas the TGA binding site, such as TGACG-motif and as-1 are unique to rice and Arabidopsis, respectively. The CGTCA-motif and TGACG-motif are present in all WRKY, MADS-box and MYB TF family genes except in the OsMYB50 gene. The MYB binding sites are found in WRKY and MYB genes, with high occurrence in the MYB genes. Other stress-related elements are found in rice genes that include the oxidative stress-responsive element (ARE) and light stress (I-box, Box II and LTR). The elicitor responsive element (W-box), light stress (GT1-motif and GATA-motif) and defense response (G-box) were consistently present in all Arabidopsis genes (Fig. 7). The orthologous rice and Arabidopsis gene pairs showed common CRE function despite displaying diversity in CRE identities and numbers. The annotation of CREs function involved in the development activities, hormone response and abiotic/biotic stress are compared among the orthologous gene pairs (Table 2).

In silico analysis of co-expression and functional similarity between Arabidopsis and rice orthologous gene pairs. Co-expression analysis was conducted on the 19 Arabidopsis and 18 rice orthologous genes identified in the previous analysis where the expression datasets were retrieved from PLANEX (planex.plantbioinformatics.org). The correlation values (r) among the WRKY, MADS-box and MYB genes in Arabidopsis and rice were ranked as follows: (i) poor; $r<0.20$, (ii) fairly moderate; $r=0.2-0.4$, (iii) fairly strong; $\mathrm{r}>0.4-0.6$ and (iv) strong; $r>0.6-0.8$. The average positive correlation within the Arabidopsis and rice network were 0.212 and 0.160 , respectively. The negative correlation of the Arabidopsis network $(\mathrm{r}=-0.248)$ was much stronger than the rice network $(\mathrm{r}=-0.084)$. In Arabidopsis, AtMYB4R1 showed the strongest correlation $(\mathrm{r}=0.465$, fairly strong) with MADS-box (AtAGL65), MYB (AtMYB103, AtMYB91, AtMYB5 and AtMYBCDC5) and WRKY (AtWRKY65, AtWRKY9, AtWRKY44, AtWRKY55 and AtWRKY43) transcription factor (TF) genes. For rice TFs, OsMYB46 showed the strongest correlation with OsMYB13, OsMYB19, OsWRKY13, OsWRKY17, OsWRKY22, OsWRKY23, OsWRKY32 and OsWRKY119 shown at $\mathrm{r}=0.827$ (Fig. 8).

The occurrence of possible functional similarity between Arabidopsis and rice orthologous genes were compared on their co-expression networks using the Kappa statistics retrieved from PLANEX (Table 3). Kappa ( $k$ ) score $=1$ denotes a perfect functional similarity between networks ${ }^{35,38}$. A $k$-score $>0$ is assumed significantly similar, whilst $k$-score $=0$ denotes no significant similarity ${ }^{35,38}$. Eleven Arabidopsis-rice orthologous genes were accounted for $69 \%$ of the total genes $(k$-score $=0.2-0.4)$ that showed fair functional similarity, followed by three genes $(19 \%)$ and two genes $(13 \%)$ of poor $(k$-score $=>0.0$ to 0.2$)$ and moderate $(k$-score $=0.4$ to 0.6$)$ functional similarity, respectively. The OsWRKY32-AtWRKY9 and OsMADS68-AtAGL65 orthologous pairs were highly significant with a $k$-score of 0.44 and 0.50 , respectively. 


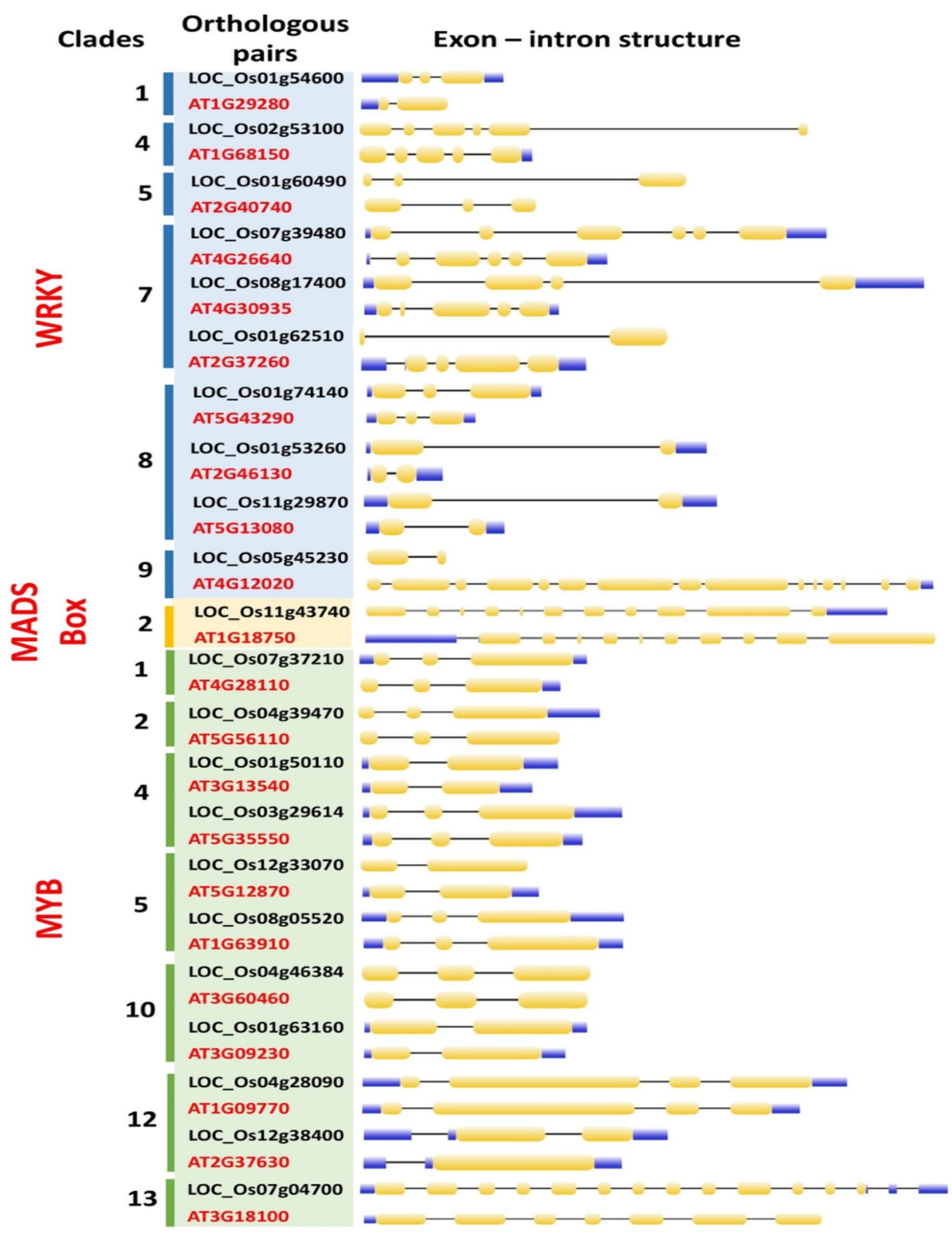

Figure 5. Exon-intron structure of Arabidopsis and rice WRKY (blue column), MADS-box (yellow column) and MYB (green column), orthologous gene pairs displayed according to clade numbers in their TF familyphylogenetic tree. The exon-intron structure is described as following: the yellow rectangles and grey lines denote exons and introns, respectively whilst the blue boxes represents the untranslated regions (UTRs).

\section{Discussion}

Over the years, natural and human activities have caused significant changes to the global environment. Climate change, decrease in arable land, increase in $\mathrm{CO}_{2}$ concentration, declining water availability, drought and high salinity had set major challenges to agricultural systems, worldwide. The quest for yield and productivity is becoming increasingly challenging with a continuum decline in plant stress resistance. Plants are complex multicellular organisms with highly flexible adaptivity to adverse conditions such as the exposure to abiotic and biotic factors that trigger various responses governed by complex regulatory mechanism i.e. the transcriptional 


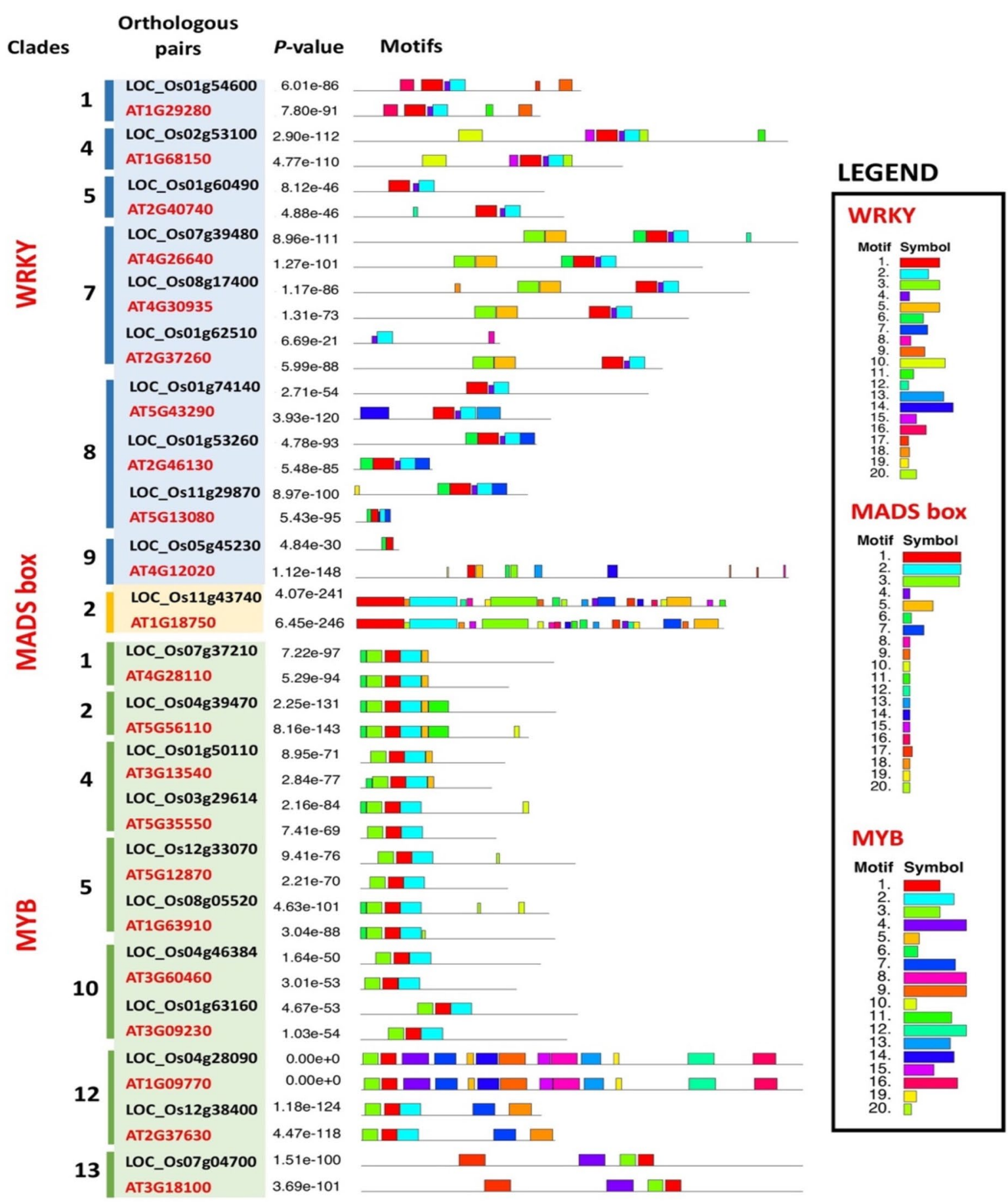

Figure 6. Distribution pattern of conserved motifs in Arabidopsis and rice WRKY, MADS-box and MYB orthologous genes, identified by MEME web server. Orthologous gene pairs are presented by transcription factor (TF) families: column blue; WRKY, column yellow; MADS-box and column green; MYB. The $p$-values are significant at 0.05 . Motif distribution includes different coloured boxes, each represent a unique numbered motif as indicated in the legend. The width differences among the boxes represents the motif length.

regulation ${ }^{39}$ and through gene expression, they respond to these changes by either activating or repressing the expression of the downstream genes ${ }^{40,41}$.

Transcription factors (TFs) are deployed as the master key regulators in plant growth and development, and defense-related responses. The WRKY, MADS-box and MYB are major TF families that regulate various aspects of plant development through specificity and/or crosstalk regulation between different TFs; growth and developmental processes ${ }^{42}$, and biotic and abiotic stress responses ${ }^{35,43,44}$. Cis-acting regulatory elements (CREs) at the binding site or near to the structural genes interact with TFs to control the expression of the corresponding 


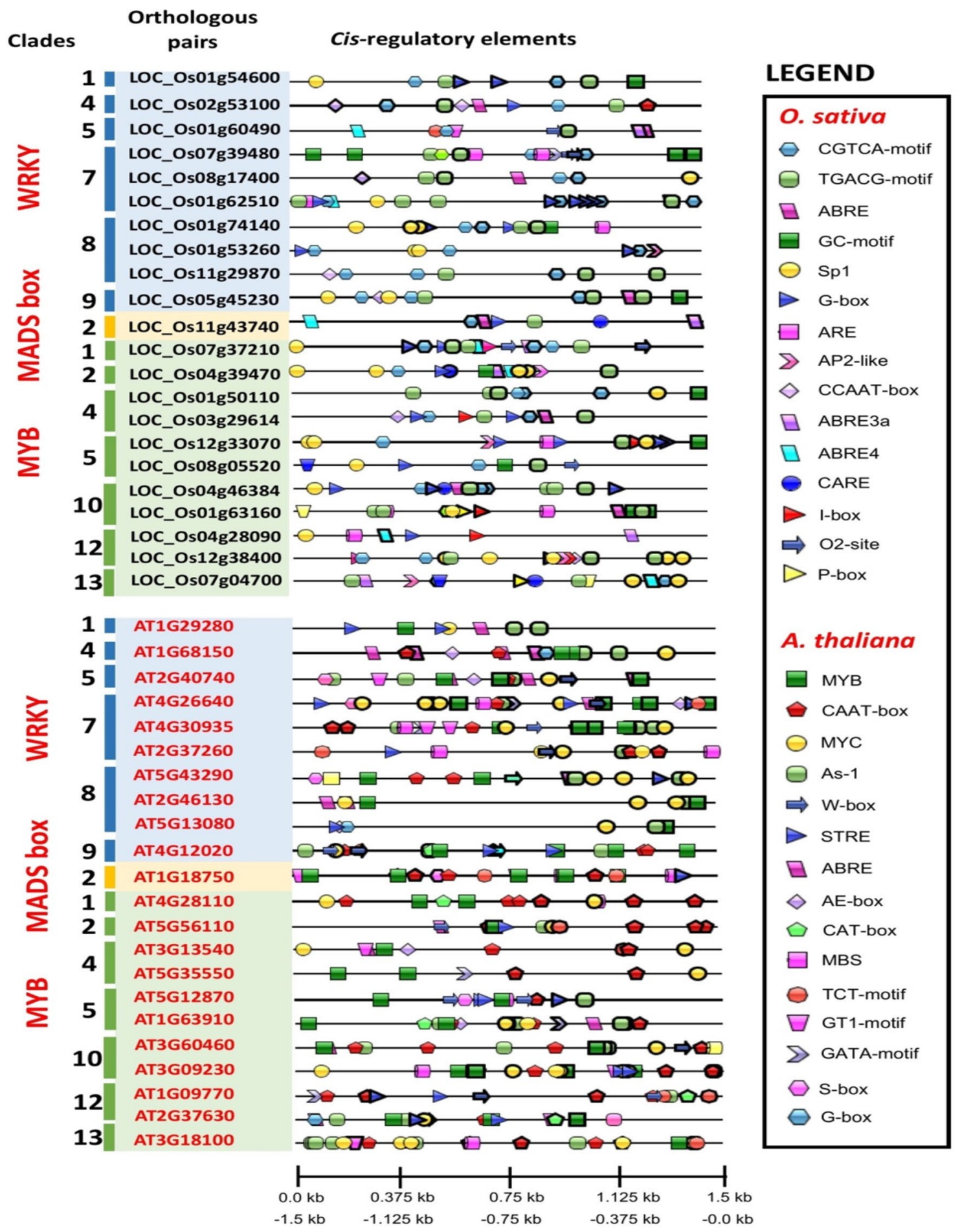

Figure 7. Distribution of the cis-regulatory elements (CRE) in the $1.5 \mathrm{~kb}$ promoter region of Arabidopsis and rice WRKY, MADS box and MYB orthologous genes as identified by PlantCARE and visualized using the IBS software (http://ibs.biocuckoo.org). The CREs are denoted by in different shapes and colours. Each CRE is drawn as following: (i) thick black line for the reverse strand and (ii) thin black line for the forward strand.

genes. The promoters present at the upstream of a gene encoded region contain numerous CREs which are unique to various proteins involved in the transcription initiation and regulation ${ }^{40,45}$. The CREs have been reported to display diverse functions associated with biotic and abiotic components: pathogen and wound responsive, light and phytohormone responsive. Studies on cis-regulatory elements (CREs) are important to further understand the plant defense responses to abiotic and biotic stresses ${ }^{38}$.

In this study, the Arabidopsis and rice WRKY, MADS-box and MYB TF genes showed a similar TF-family abundance level. Although the rice genome size is larger than Arabidopsis's, the number of TF genes in both 


\begin{tabular}{|c|c|c|c|c|c|}
\hline \multirow[b]{2}{*}{ Clade } & \multirow[b]{2}{*}{ Gene identifier } & \multirow[b]{2}{*}{ Name } & \multicolumn{3}{|l|}{ CRE function } \\
\hline & & & Development & Hormone response & Abiotic/biotic stress \\
\hline \multirow[t]{2}{*}{2} & LOC_Os11g43740 & OsMADS68 & $\mathrm{N} / / \mathrm{A}$ & $\begin{array}{l}\text { CGTCA-motif, TGACG-motif, ABRE, ABRE3a, } \\
\text { ABRE4 }\end{array}$ & G-box \\
\hline & AT1G18750 & AtAGL65 & N/A & ABRE & S-box, GT1-motif, MBS, MYB, STRE, TCT-motif \\
\hline \multirow[t]{2}{*}{1} & LOC_Os07g37210 & OsMYB102 & C-box, O2-site & $\begin{array}{l}\text { ABRE3a, ABRE4, CGTCA-motif, TGACG-motif, } \\
\text { O2-site }\end{array}$ & C-box, Sp1 \\
\hline & AT4G28110 & AtMYB41 & CAT-box & N/A & MBS, MYC, MYB \\
\hline \multirow{2}{*}{2} & LOC_Os04g39470 & OsMYB80 & Motif I, AP-2 like & ABRE3a, ABRE4, CGTCA-motif, TGACG-motif & G-box, GC-motif, Sp1 \\
\hline & AT5G56110 & AtMYB80 & As-1 & ABRE, As-1 & MYB, MYC, STRE, TCT-motif, W-box \\
\hline \multirow{4}{*}{4} & LOC_Os01g50110 & OsMYB13 & N/A & CGTCA-motif, TGACG-motif & GC-motif, Sp1 \\
\hline & AT3G13540 & AtMYB5 & N/A & ABRE & TCT-motif, MYC, AE-box, GT1-motif, MYB \\
\hline & LOC_Os03g29614 & OsMYB46 & N/A & ABRE, CGTCA-motif, TGACG-motif & G-box, I-box, CCAAT-box \\
\hline & AT5G35550 & AtMYB123 & N/A & N/A & MYC, GATA-motif, MYB \\
\hline \multirow{4}{*}{5} & LOC_Os12g33070 & OsMYB122 & AP-2 like & CGTCA-motif, TGACG-motif & ARE, G-box, GC-motif, I-box, Sp1 \\
\hline & AT5G12870 & AtMYB46 & As-1 & As-1 & S-box, MBS, MYB, STRE, W-box \\
\hline & LOC_Os08g05520 & OsMYB93 & O2-site & CGTCA-motif, TGACG-motif, O2-site & G-box, GC-motif, Spl \\
\hline & AT1G63910 & AtMYB103 & As-1, CAT-box & ABRE, As-1 & AE-box, GATA-motif, MYB, MYC \\
\hline \multirow{4}{*}{10} & LOC_Os04g46384 & OsMYB58 & N/A & ABRE, CGTCA-motif, TGACG-motif & G-box, Sp1 \\
\hline & AT3G60460 & AtMYB125 & As-1 & ABRE, As-1 & W-box, MYC, MYB, sbp-CMA1c \\
\hline & LOC_Os01g63160 & OsMYB19 & GCN4_motif & ABRE, CGTCA-motif, TGACG-motif & ARE, GC-motif, I-box, LTR, P-box, Sp1 \\
\hline & AT3G09230 & AtMYB1 & As-1 & As-1 & AE-box, GT1-motif, MBS, MYB, MYC, STRE \\
\hline \multirow{4}{*}{12} & LOC_Os04g28090 & OsMYB50 & N/A & ABRE3a, ABRE4 & ARE, G-box, P-box, Sp1 \\
\hline & AT1G09770 & AtMYBCDC5 & As-1, CAT-box & As-1 & GATA-motif, STRE, TCT-motif, W-box \\
\hline & LOC_Os12g38400 & OsMYB125 & C-box, AP-2 like & CGTCA-motif, TGACG-motif & C-box, CCAAT-box, G-box, Sp1 \\
\hline & AT2G37630 & AtMYB91 & As-1, CAT-box & ABRE, JERE & AE-box, MYB, MYC, STRE \\
\hline \multirow{2}{*}{13} & LOC_Os07g04700 & OsMYB87 & AP-2 like & ABRE3a, ABRE4, CGTCA-motif, TGACG-motif & LTR, P-box, Sp1 \\
\hline & AT3G18100 & AtMYB4R1 & As-1 & As-1 & GT1-motif, MBS, MYB, MYC, TCT-motif \\
\hline \multirow{2}{*}{1} & LOC_Os01g54600 & OsWRKY13 & N/A & CGTCA-motif, TGACG-motif & GC-motif, G-box \\
\hline & AT1G29280 & AtWRKY65 & As-1 & ABRE, As-1 & MYB, MYC, STRE \\
\hline \multirow{2}{*}{4} & LOC_Os02g53100 & OsWRKY32 & N/A & ABRE, CGTCA-motif, TGACG-motif & G-box, CCAAT-box, Sp-1 \\
\hline & AT1G68150 & AtWRKY9 & As-1 & ABRE, As-1 & AE-box, G-box, MYB, MYC \\
\hline \multirow{2}{*}{5} & LOC_Os01g60490 & OsWRKY22 & O2-site & $\begin{array}{l}\text { ABRE, ABRE3a, ABRE4, CGTCA-motif, TGACG- } \\
\text { motif, O2-site }\end{array}$ & Box II \\
\hline & AT2G40740 & AtWRKY55 & As-1, CAT-box & ABRE, As-1 & AE-box, S-box, GT1-motif, MYB, MYC, W-box \\
\hline \multirow{6}{*}{7} & LOC_Os07g39480 & OsWRKY87 & GCN4_motif, O2-site & CGTCA-motif, TGACG-motif, O2-site & ARE, GC-motif \\
\hline & AT4G26640 & AtWRKY20 & As-1 & ABRE, As-1 & $\begin{array}{l}\text { AE-box, S-box, G-box, GATA-motif, MBS, MYB, } \\
\text { MYC, STRE, TCT-motif, W-box }\end{array}$ \\
\hline & LOC_Os08g17400 & OsWRKY89 & O2-site & ABRE, CGTCA-motif, TGACG-motif, O2-site & CCAAT-box, Spl \\
\hline & AT4G30935 & AtWRKY32 & As-1 & As-1 & GATA-motif, GT1-motif, MBS, MYB, MYC, W-box \\
\hline & LOC_Os01g62510 & OsWRKY119 & N/A & ABRE3a, ABRE4, CGTCA-motif, TGACG-motif & ARE, G-box, GC-motif, Sp1 \\
\hline & AT2G37260 & AtWRKY44 & As-1 & As-1 & MBS, MYC, STRE, TCT-motif, W-box \\
\hline \multirow{6}{*}{8} & LOC_Os01g74140 & OsWRKY17 & N/A & CGTCA-motif, TGACG-motif & ARE, G-box, Sp1, GC-motif \\
\hline & AT5G43290 & AtWRKY49 & As-1 & ABRE, As-1 & S-box, DRE core, Gap-box, MYB, MYC, STRE \\
\hline & LOC_Os01g53260 & OsWRKY23 & AP-2 like & CGTCA-motif, TGACG-motif & G-box, Sp1 \\
\hline & AT2G46130 & AtWRKY43 & As-1 & ABRE, As-1 & MYB, MYC \\
\hline & LOC_Os11g29870 & OsWRKY72 & N/A & CGTCA-motif, TGACG-motif & CCAAT-box \\
\hline & AT5G13080 & AtWRKY75 & As-1 & As-1 & MYC, MYB, AE-box, G-box, STRE \\
\hline \multirow{2}{*}{9} & LOC_Os05g45230 & OsWRKY58 & N/A & ABRE, CGTCA-motif, TGACG-motif & CCAAT-box, GC-motif, Sp1 \\
\hline & AT4G12020 & AtWRKY19 & As-1, CAT-box & As-1 & DRE core, MYB, MYC, STRE, W-box \\
\hline
\end{tabular}

Table 2. Comparison of plant development, hormone and stress-responsive cis-regulatory elements (CREs) in the promoter regions of Arabidopsis and rice WRKY, MADS-box, and MYB orthologous gene pairs.

species were similar. Phylogenetic trees built on a collated rice and Arabidopsis WRKY, MADS-box and MYB TF family members were each divided into 10, 7 and 14 clades, respectively. The findings suggest that MYB TF family is the most diverse family, followed by WRKY and the MADS-box, being the least diverse TF family. Generally, both WRKY and MYB TF members were much closely related to one other in comparison to MADS-box members. In the WRKY- and MYB- specific phylogenetic tree, both the Arabidopsis and rice genes 
(A)

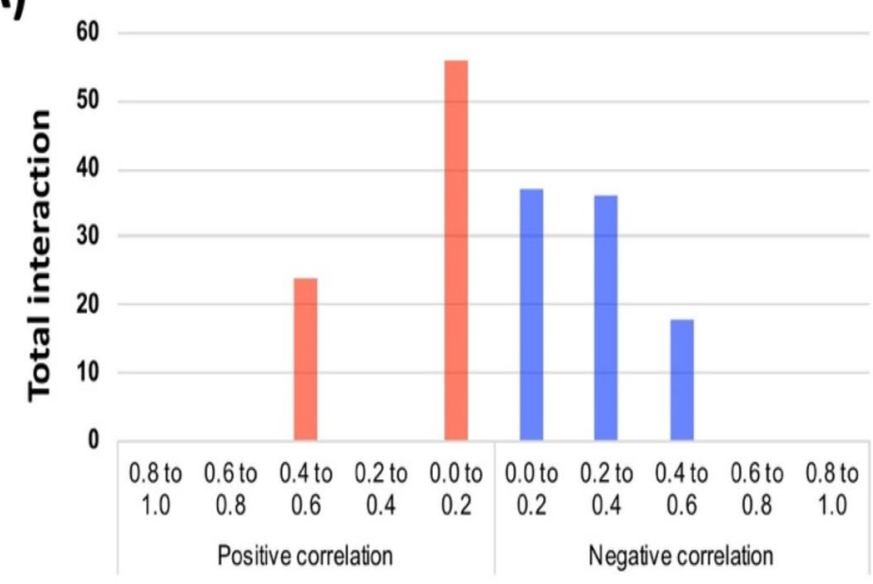

r-value

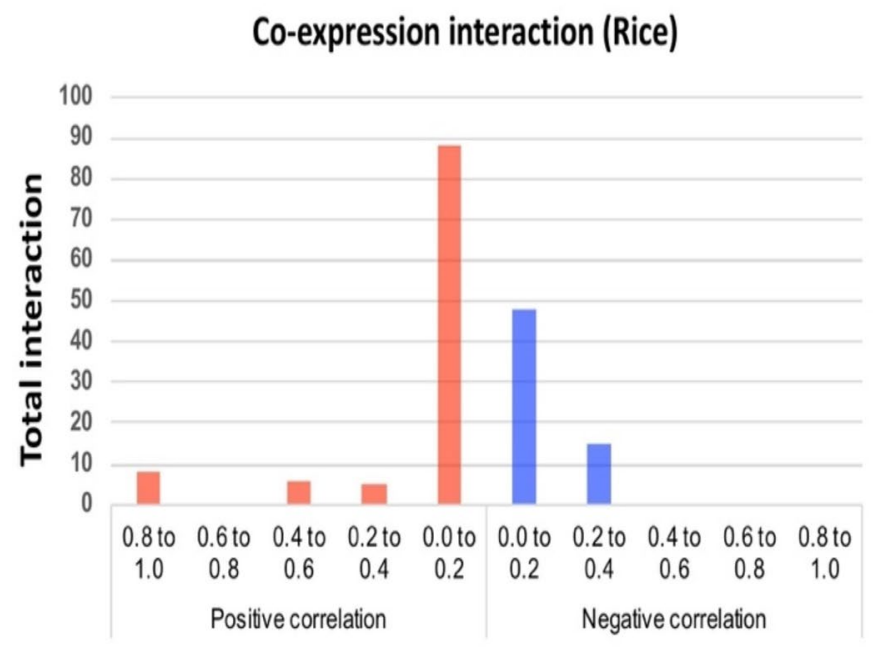

r-value

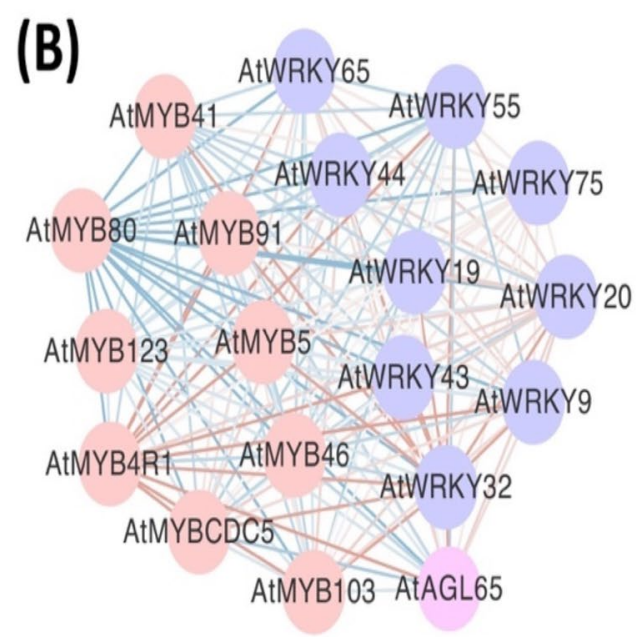

r-value
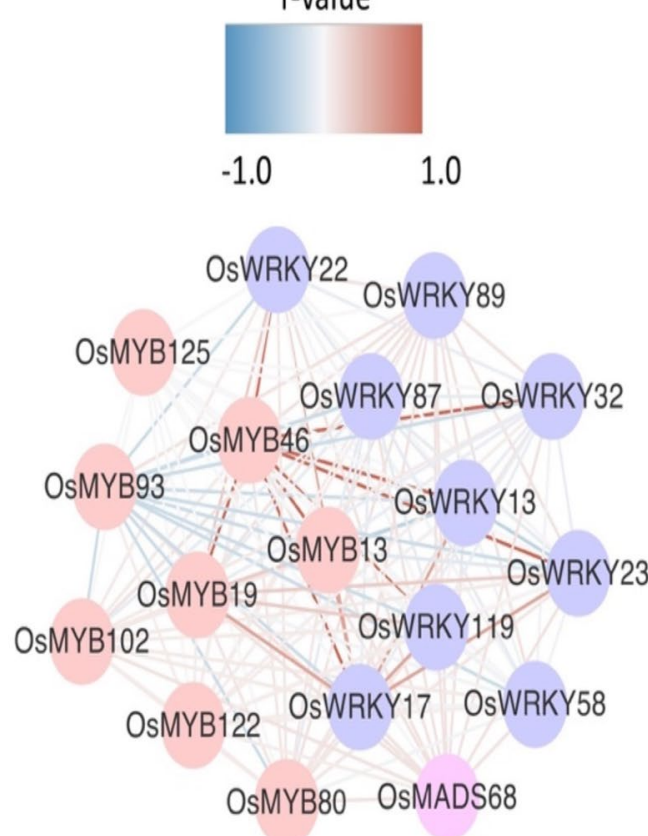

Figure 8. Gene co-expression network of Arabidopsis and rice WRKY, MADS-box and MYB orthologous genes. (A) Frequencies of co-expression interactions identified by PLANEX. Increasing r-values show stronger positive correlation and vice versa. (B) Co-expression network comprised of nodes, represent genes, different node colour s indicate unique transcription factor family (red node $=$ MYB, blue node $=$ WRKY and purple node $=$ MADS-box) and edges indicate positive (red lines) and negative (blue lines) correlations.

were present in virtually all clades. In contrast, MADS-box specific-phylogenetic tree, very few clades showed a representation of rice and Arabidopsis; clades were dominated by a single species, either the Arabidopsis or rice (Fig. 2). Ortholog genes are similar genes with the same gene function that may have arisen from speciation events. A relatively higher number of orthologous gene pairs observed in the Arabidopsis-rice WRKY and MYB TF families may explain the existence of ancestral relationships between Arabidopsis and rice before divergence during evolution (Figs. 1 and 3). Chromosomal distribution of orthologous WRKY and MYB genes in rice and Arabidopsis showed no apparent pattern. However, it is noteworthy to mention that most of the orthologous genes were distributed within the single arms of the chromosomes (Fig. 4).

Gene structure analysis imparts understanding into evolutionary processes such as duplication events ${ }^{46}$. In this study, the three different TF orthologous gene families from Arabidopsis and rice displayed various exon and intron numbers, implying possible roles in diversification events of the two Angiosperms. For instance, the rice OsWRKY13 gene consists of three exons, whilst its counterpart orthologous pair, the Arabidopsis AtWRKY65 contains two exons only. These results suggest that some of the TF family genes may have undergone loss of introns during the evolutionary processes and cause subsequent functional differences in rice and Arabidopsis. Most of the Arabidopsis-rice orthologous gene pairs under the WRKY and MYB TF family consist of similar exon numbers, and thus, implies similar gene function acquirement during stable evolution ${ }^{47}$. The number of 


\begin{tabular}{|l|l|l|l|l|l|l|}
\hline Rice & Arabidopsis & \multirow{2}{*}{ Kappa statistics } \\
\hline Gene ID & Name & Probe ID & Gene ID & Name & Probe ID & (1) \\
\hline LOC_Os11g43740 & OsMADS68 & OsAffx.19355.1.S1_at & AT1G18750 & AtAGL65 & 261423_at & 0.500029866483831 \\
\hline LOC_Os07g37210 & OsMYB102 & Os.3390.1.S1_at & AT4G28110 & AtMYB41 & 253851_at & 0.236403501449661 \\
\hline LOC_Os04g39470 & OsMYB80 & OsAffx.14205.1.S1_at & AT5G56110 & AtMYB80 & 248051_at & 0.162086262661477 \\
\hline LOC_Os01g50110 & OsMYB13 & Os.55528.1.S1_at & AT3G13540 & AtMYB5 & 256985_at & 0.342306085866936 \\
\hline LOC_Os03g29614 & OsMYB46 & Os.56985.1.S1_a_at & AT5G35550 & AtMYB123 & 249704_at & 0.355608075400779 \\
\hline LOC_Os12g33070 & OsMYB122 & OsAffx.19945.2.S1_at & AT5G12870 & AtMYB46 & 250322_at & 0.122141588277817 \\
\hline LOC_Os08g05520 & OsMYB93 & Os.49830.1.S1_at & AT1G63910 & AtMYB103 & 260326_at & 0.316581470795973 \\
\hline LOC_Os12g38400 & OsMYB125 & Os.12994.1.S1_at & AT2G37630 & AtMYB91 & 267157_at & 0.348381644725455 \\
\hline LOC_Os01g54600 & OsWRKY13 & Os.2160.2.S1_x_at & AT1G29280 & AtWRKY65 & 260882_at & 0.293798964280973 \\
\hline LOC_Os02g53100 & OsWRKY32 & OsAffx.12620.1.S1_at & AT1G68150 & AtWRKY9 & 260432_at & 0.439323046291688 \\
\hline LOC_Os01g60490 & OsWRKY22 & OsAffx.23871.1.S1_at & AT2G40740 & AtWRKY55 & 266052_at & 0.309001957815947 \\
\hline LOC_Os07g39480 & OsWRKY87 & Os.18862.1.S1_at & AT4G26640 & AtWRKY20 & 253983_at & 0.263336526419911 \\
\hline LOC_Os08g17400 & OsWRKY89 & Os.27818.1.S1_at & AT4G30935 & AtWRKY32 & 253603_at & 0.206776541657964 \\
\hline LOC_Os01g62510 & OsWRKY119 & OsAffx.9554.1.S1_at & AT2G37260 & AtWRKY44 & 265954_at & 0.185878341728013 \\
\hline LOC_Os01g53260 & OsWRKY23 & Os.30386.1.S1_at & AT2G46130 & AtWRKY43 & 266597_at & 0.372919466881054 \\
\hline LOC_Os05g45230 & OsWRKY58 & OsAffx.27315.1.S1_at & AT4G12020 & AtWRKY19 & 254852_at & 0.208021020730278 \\
\hline
\end{tabular}

Table 3. Functional similarity between the Arabidopsis and rice WRKY, MADS-box and MYB orthologous gene-pairs. The co-expression datasets are retrieved and analyzed using Kappa statistics from PLANEX.

proteins with motifs identified in the WRKY TF family was comparable to the MYB TF family; 20-22 proteins. The MADS-box TF family contained only two protein sequences with motifs (Fig. 6). The disparity between the WRKY and MYB TF families over the MADS-box TF family could be implicated in the functional differences between these TF families. The MADS-box are highly involved in plant growth and development in comparison to WRKY and MYB TF families which are actively responsive to biotic and abiotic responses. Similar types of motifs were identified in all three TF families, however, the motif and CRE distribution displayed a similar trend by the TF family suggesting the functional niche unique to each TF family.

Motif distributions are conserved between the orthologous gene pairs that share a common clade. Each specific motif present in the orthologous genes corresponds to a specific protein function. For example, WRKY genes with a DNA-binding domain were mainly enriched within motif 1-3 and 5. MYB genes enriched with motif 1-4 correspond to Myb-like DNA-binding domain and MADS-box genes with an abundant number of motif 1 , motif 3 and motif 5 correspond to DNA-binding and dimerisation domain, K-box region and connexin4, respectively. In general, WRKY and MYB orthologous genes show motif abundance and diversity to a major extent. It is also noteworthy to observe the impact of motif loss in the orthologous gene pairs. As such, the rice OsWRKY58 gene lacks motifs 5, 6, 9, 10,13,14, 15 and 18 in comparison to its orthologous pair, which is the Arabidopsis AtWRKY19 gene. These differences may imply the occurrence of the OsWRKY58 gene functional divergence with the AtWRKY19 gene.

The CRE analysis of Arabidopsis and rice WRKY, MADS-box and MYB genes showed functional involvement in stress-related, phytohormone-related and plant development-related activities. All Arabidopsis and rice genes contain a combination of different CREs except for the following orthologous pairs which contain a phytohormone-related ABRE motif: OsMADS68-AtAGL65 (clade 2, MADS-box TF), OsMYB58-AtMYB125 (clade 10, MYB TF) and OsWRKY22-AtWRKY55 (clade 5, WRKYT F). The OsWRKY32-AtWRKY9 orthologous pair share both ABRE and G-box element motifs. Previous studies showed the role of G-box as a stress-responsive element against pathogen ${ }^{48}$, in phytohormone like abscisic acid (ABA) and jasmonic acid (JA) signalling regulator, and favours reactive oxygen species (ROS) burst under environmental stress ${ }^{47,49}$. Additionally, ABA responsive element (ABRE) also acts as a positive regulator of ABA signalling under saline and drought conditions ${ }^{47,50}$. Phytohormone-related elements (CGTCA-motif and TGACG-motif) abundantly present in rice genes suggest its crucial function in JA-responsiveness. The TGACG-motif and As-1 elements are both known as TGA elements. Interestingly, TGACG-motif was predominantly found in rice genes and As-1 element in Arabidopsis genes, mainly. Our findings showed an apparent divergence of stress-related elements in rice and Arabidopsis. The CREs that are unique to rice genes are Sp1, ARE and GC-motif. On the other hand, MYB, MYC, STRE and W-box motifs are unique to the Arabidopsis gene. ARE (Anaerobic responsive elements) consisting of GC and GT motifs act as an oxidative responsive element. Previous studies showed that the rice genome contains higher GC motifs than in Arabidopsis ${ }^{47,51}$.

An ongoing duplication event within plant species may had led to the divergence of the WRKY, MADSbox and MYB TF families. Apparent gain and loss in gene structures were evident within each TF family. Co-expression network analysis revealed a moderately fair $(\mathrm{r}=0.2-0.4)$ interaction in Arabidopsis and poor interaction $(\mathrm{r}=>0-0.2)$ in rice. OsMYB46 gene in rice encodes the transcriptional regulation of secondary wall biosynthesis. Rice co-expression network analysis has shown a strong association of the OsMYB46 gene with lignin biosynthetic transcription factors (OsMYB13 and $O s M Y B 19)^{52}$, and rice resistance to blast and bacterial blight encoding OsWRKY $22^{53}$, OsWRKY $13^{54}$ and OsWRKY $23^{55}$ genes. These findings suggest that both MYB 
and WRK TF family genes are switched on to orchestrate SA- and JA- mediated signalling pathways during the pathogen attack.

The functional similarities between WRKY, MADS-box and MYB genes within Arabidopsis and rice was measured and compared against each other via the co-expression network analysis. Two independent Arabidopsis and rice co-expression networks were about similar size as indicated by the total number of nodes (number of genes); 19 in Arabidopsis and 18 in the rice co-expression network. In each co-expression network, all three different WRKY, MADS-box and MYB genes showed positive and negative correlations to a considerable extent. Interestingly, the hub gene denotes as the gene with the most number of interactions belongs to the MYB TF family in both Arabidopsis and rice co-expression networks.

The functional similarities of Arabidopsis and rice orthologous gene- pairs were detected at significant $\mathrm{k}$-scores ${ }^{38}$. Previously studies using co-expression networks analysis have functionally characterized several genes, i.e. the Arabidopsis AtAGL65 gene that regulates pollen tube growth and maturation ${ }^{56}$, and OsMADS68 that regulates the downstream OsCPK21 gene during anther development in rice ${ }^{57}$. The OsMYB80-AtMYB80, riceArabidopsis orthologous gene pair is functionally conserved as the positive regulators of pollen development ${ }^{58,59}$. Meanwhile, the Arabidopsis AtWRKY9 gene was shown to be induced in response to pathogen-associated molecular patterns (PAMP) ${ }^{52}$, and the rice OsWRKY32 gene has been activated during rice blast pathogen, Magnoporthae oryzae pathogenesis ${ }^{60}$. Based on the expression profiles, Arabidopsis AtWRKY43 gene showed close association with the pathogen defense transcription factor, the rice OsWRKY23 gene ${ }^{55,61}$. The discovery of stress-related genes and their association with the Arabidopsis and rice WRKY, MADS-box and MYB orthologous genes offers a basis for future biotechnology and breeding studies aimed to enhance plant stress responses.

Feeding more than half the world population, rice is a premier staple food worldwide, especially among the majority of Asians. Rice yield improvement has been a key breeding objective as farming and subsequent productivity are affected by numerous factors such as soil fertility, abiotic stressors (salinity, drought, heat and cold) and susceptibility to a wide range of diseases. The present-day rice breeding strategies have evolved tremendously. From conventional breeding to breeding by design, the identification of candidate desirable genes is a core component to kickstart any breeding programmes. Improvement of complex traits controlled by multiple genes with each displaying a relatively small effect had led to trait-based selections that are unfavourably related ${ }^{62}$. As a result, the current pace of rice breeding does not meet the breeding objectives designed for the development of climate-resilient, fit and adaptive, and resource-use efficient cultivars.

Gene similarities are key aspect of gene function. Gene data sets which includes the gene expression and gene co-expression networks elucidate associated functions between genes across and within the plant kingdoms. The overall functional similarity between two genes requires multi-aspect considerations. Although both rice and Arabidopsis are two important model plant organisms subjected to different research pace, the latter is much more thoroughly investigated and functionally described in comparison to rice. In addition, most gene function association studies performed are projected on Arabidopsis to better understand the any given plant organism of interest. In this study, the Arabidopsis and rice TF families are comparatively evaluated to gain multi-dimensional information on the WRKY, MADS-box and MYB gene pattern of distribution, structure and function.

In the 'breeding by design' technique such as the target chromosome-segment substitution ${ }^{63}$, mapping of loci governing agronomically desirable traits serves as the pre-requisite step. Under this technique, information on the desirable gene loci along their interrelated functional roles is crucial to accomplish a successful breeding programme. Ultimately, using transcription factor genes, the present findings offer a knowledge base to facilitate efficient selection of desirable genes as TF genes among the different families (WRKY, MADS-box and MYB) displaying inter-relations with each other. In parallel, current findings enables manipulation of biologically important multi-functional TF genes governing rice stress responses and developmental processes. Rice improvement guards global food security, and thus, the production of resilient planting materials could be facilitated and accelerated in breeding programmes catered for rapid development of rice varieties.

\section{Conclusions}

Plant growth and development, and environmental responses are key targets for manipulation in biotechnology and breeding programmes. This study investigated 172 WRKY, 100 MADS-box and 266 MYB TF genes in Arabidopsis and rice. Twenty-two Arabidopsis-rice orthologous gene pairs were identified from the WRKY, MADS-box and MYB TF family, and their exon-intron distribution along the motif compositions are mostly similar and conserved. The majority of the WRKY, MADS-box and MYB genes in Arabidopsis and rice showed specific interaction with abiotic/biotic and phytohormone responsiveness elements. Further, the co-expression interaction among the WRKY, MADS-box and MYB genes between Arabidopsis and rice illustrated a similar trend based on the average correlation measurement. The functional similarity of co-expression data comprised of orthologous genes indicates their important roles in pollen development, hormone-mediated and defense response to the pathogen. The orthologous genes identified in this study informs the selection of genes governing the conserved regulatory module of defense and development in rice and Arabidopsis.

Received: 23 June 2021; Accepted: 21 September 2021

Published online: 04 October 2021

\section{References}

1. Hao, L. Y. et al. Genome-wide identification and comparative analysis of drought related genes in roots of two maize inbred lines with contrasting drought tolerance by RNA sequencing. J. Integr. Agric. 19(2), 449-464 (2020).

2. Mishra, P. et al. In silico mining of WRKY TFs through Solanum melongena L. and Solanum incanum L. transcriptomes and identification of SiWRKY53 as a source of resistance to bacterial wilt. Plant Gene 26, 1278 (2021). 
3. Balaguer, M. A. D. L. et al. Predicting gene regulatory networks by combining spatial and temporal gene expression data in Arabidopsis root stem cells. Proc. Natl. Acad. Sci. U. S. A. 114(36), E7632-E7640 (2017).

4. Joshi, R. et al. Transcription factors and plants response to drought stress: Current understanding and future directions. Front Plant Sci. 7, 1029. https://doi.org/10.3389/fpls.2016.01029 (2016).

5. Srivastava, R. et al. Comparative genome-wide analysis of WRKY transcription factors in two Asian legume crops: Adzuki bean and Mung bean. Sci. Rep. 8(1), 16971 (2018).

6. Wu, K. L., Guo, Z. J., Wang, H. H. \& Li, J. The WRKY family of transcription factors in rice and Arabidopsis and their origins. DNA Res. 12(1), 9-26 (2005).

7. Rushton, P. J., Somssich, I. E., Ringler, P. \& Shen, Q. J. WRKY transcription factors. Trends Plant Sci. 15(5), 247-258 (2010).

8. Yanhui, C. et al. The MYB transcription factor superfamily of Arabidopsis: Expression analysis and phylogenetic comparison with the rice MYB family. Plant Mol. Biol. 60(1), 107-124 (2006).

9. Li, C., Ng, C. K. Y. \& Fan, L. M. MYB transcription factors, active players in abiotic stress signaling. Environ. Exp. Bot. 114, 80-91 (2015).

10. Ogatallz, K. et al. The cavity in the hydrophobic core of Myb DNA-binding domain is reserved for DNA recognition and transactivation. Nat. Struct. Biol. 3, 178-187 (1996).

11. Jia, L., Clegg, M. T. \& Jiang, T. Evolutionary dynamics of the DNA-binding domains in putative R2R3-MYB genes identified from rice subspecies indica and japonica genomes. Plant Physiol. 134, 575-585 (2004).

12. Leng, B. et al. Heterologous expression of the Limonium bicolor MYB transcription factor LbTRY in Arabidopsis thaliana increases salt sensitivity by modifying root hair development and osmotic homeostasis. Plant Sci. 302, 110704 (2021).

13. Initiative, T. A. G. Analysis of the genome sequence of Arabidopsis thaliana. Nature 408(6814), 796-815 (2000).

14. Wixon, J. Arabidopsis thaliana. Int. J. Genomics 2(2), 91-98 (2001).

15. Jin, J. et al. PlantTFDB 4.0: Toward a central hub for transcription factors and regulatory interactions in plants. Nucl. Acids Res. 45, gkw982 (2016).

16. Tiwari, P. et al. Auxin-salicylic acid cross-talk ameliorates OsMYB-R1 mediated defense towards heavy metal, drought and fungal stress. J. Hazard Mater. https://doi.org/10.1016/j.jhazmat.2020.122811 (2020).

17. Park, D. Y. et al. The MYB- related transcription factor RADIALIS-LIKE3 (OsRL3) functions in ABA-induced leaf senenscence and salt sensitivity in rice. Environ. Exp. Bot. 156, 86-95 (2018).

18. Wang, P. et al. OsWRKY28 regulates phosphate and arsenate accumulation, root system, architecture and fertility in rice. Front. Plant Sci. 9, 1330 (2018).

19. Dai, X., Wang, Y. \& Zhang, W. H. OsWRKY74, a WRKY transcription factor, modulates tolerance to phosphate starvation in rice. J. Exp. Bot. 67(3), 947-960 (2016).

20. Chen, H. et al. OsMADS27 regulates the root development in a NO3-dependent manner and modulates the salt tolerance in rice (Oryza sativa L.). Plant Sci. 277, 20-32 (2018).

21. Yuan, F., Xu, Y., Leng, B. \& Wang, B. beneficial effects of salt on halophyte growth: morphology, cells and genes. Open Life Sci. 14, 191-200 (2019).

22. Smaczniak, C., Immink, R. G., Angenent, G. C. \& Kaufmann, K. Developmental and evolutionary diversity of plant MADS-domain factors: insights from recent studies. Development 139, 3081-3098 (2012).

23. Goodstein, D. M. et al. Phytozome: A comparative platform for green plant genomics. Nucl. Acids Res. 40, D1178-D1186 (2012).

24. Thompson, J. D., Gibson, T. J. \& Higgins, D. G. Multiple sequence alignment using ClustalW and ClustalX. Curr. Protoc. Bioinform. https://doi.org/10.1002/0471250953.bi0203s00 (2002).

25. Saitou, N. \& Nei, M. The neighbor-joining method: A new method for reconstructing phylogenetic trees. Mol. Biol. Evol. 4(4), 406-425 (1987).

26. Gascuel, O. \& Steel, M. Neighbor-joining revealed. Mol. Biol. Evol. 23(11), 1997-2000 (2006).

27. Rambaut, A. FigTree v1.4.3:Molecular Evolution, Phylogenetics and Epidemiolog; 2007 Updated (2016).

28. Rhee, S. Y. et al. The Arabidopsis Information Resource (TAIR): A model organism database providing a centralized, curated gateway to Arabidopsis biology, research materials and community. Nucl. Acids Res. 31(1), 224-228 (2003).

29. Kurata, N. \& Yamazaki, Y. Oryzabase. An integrated biological and genome information database for rice. Plant Physiol. 140(1), $12-17$ (2006).

30. Wang, H., Zhao, S., Gao, Y. \& Yang, J. Characterization of dof transcription factors and their responses to osmotic stress in poplar (Populus trichocarpa)". PLoS ONE 12(1), e0170210. https://doi.org/10.1371/journal.pone.0170210 (2017).

31. Hu, B., Jin, J., Guo, A., Zhang, H. \& Luo, J. GSDS 2.0: An upgraded gene feature visualization server. Bioinformatics 31(8), 1296-1297 (2014).

32. Bailey, T. L., Johnson, J., Grant, C. E. \& Noble, W. S. The MEME suite. Nucl. Acids Res. 43, W39-W49 (2015).

33. Finn, R. D. et al. Pfam: the protein families database. Nucl. Acids Res. 42, D222-D230 (2014).

34. Lescot, M. et al. PlantCARE, a database of plant cis-acting regulatory elements and a portal to tools for in silico analysis of promoter sequences. Nucl. Acids Res. 30(1), 325-327 (2002).

35. Chen, C. et al. Heat stress yields a unique MADS box transcription factor in determining seed size and thermal sensitivity. Plant Physiol. 171(1), 606-622 (2016).

36. Yim, W. C., Yu, Y., Song, K., Jang, C. S. \& Lee, B. M. PLANEX: The plant co-expression database. BMC Plant Biol. 13(1), 83 (2013).

37. Shannon, P. et al. Cytoscape: A software environment for integrated models of biomolecular interaction networks. Genome Res. 13(11), 2498-2504 (2003).

38. Kaur, A., Pati, P. K., Pati, A. M. \& Nagpal, A. K. In-silico analysis of cis-acting regulatory elements of pathogenesis-related proteins of Arabidopsis thaliana and Oryza sativa. PLoS ONE 12(9), e0184523 (2017).

39. Maag, D., Erb, M., Köllner, D. \& Gershenzon, J. Defensive weapons and defense signals in plants: Some metabolites serve both roles. BioEssays 37(2), 167-174 (2014).

40. Ho, C. L. \& Geisler, M. Genome-wide computational identification of biologically significant Cis-regulatory elements and associated transcription factors from rice. Plants (Basel) 8(11), 441. https://doi.org/10.3390/plants8110441 (2019).

41. Mulat, M. W. \& Sinha, V. B. Identification and characterization of Dof in Tef[Eragrostis tef (Zucc.) Troetter]. using in silico approaches. Gene Rep. 19, 100590 (2020).

42. Ramamoorthy, R., Jiang, S., Kumar, N., Venkatesh, P. N. \& Ramachandran, S. A comprehensive transcriptional profiling of the WRKY gene family in rice under various abiotic and phytohormone treatments. Plant Cell Physiol. 49, 865-879. https://doi.org/ 10.1093/pcp/pcn061 (2018).

43. Wu, T. Y. et al. Crosstalk between heterotrimeric G protein-coupled signaling pathways and WRKY transcription factors modulating plant responses to suboptimal micronutrient conditions. J. Exp. Bot. 71(10), 3227-3239. https://doi.org/10.1093/jxb/eraa108 (2020).

44. Jiang, J. et al. WRKY transcription factors in plant responses to stresses. J. Integr. Plant Biol. 59(2), 86-101 (2017).

45. Mulat, M. W. \& Sinha, V. B. Distribution and abundance of CREs in the promoters depicts crosstalk by WRKYs in Tef[Eragrostic tef (Zucc) Troetter]. Gene Rep. 23, 101043 (2021).

46. Yang, A., Dai, X. \& Zhang, W. H. A R2R3-type MYB gene, OsMYB2, is involved in salt, cold, and dehydration tolerance in rice. J. Exp. Bot. 63(7), 2541-2556 (2012). 
47. Dai, X. et al. Overexpression of an R1R2R3 MYB gene, OsMYB3R-2, increases tolerance to freezing, drought, and salt stress in transgenic Arabidopsis. Plant Physiol. 143(4), 1739-1751 (2007).

48. Kong, W., Ding, L., Cheng, J. \& Wang, B. Identification and expression analysis of genes with pathogen-inducible cis-regulatory elements in the promoter regions in Oryza sativa. Rice 11(1), 52 (2018).

49. Ramegowda, V. et al. GBF3 transcription factor imparts drought tolerance in Arabidopsis thaliana. Sci. Rep. 7(1), 9148 (2017).

50. Lu, Y. et al. Genome-wide identification and expression analysis of glycine-rich RNA-binding protein family in sweet potato wild relative Ipomoea trifida. Gene 686, 177-186 (2019).

51. Ferguson, A. A. \& Jiang, N. Pack-MULEs: recycling and reshaping genes through GC-biased acquisition. Mob. Genet. Elements 1(2), 135-138 (2011).

52. Zhong, R. et al. Transcriptional activation of secondary wall biosynthesis by rice and maize NAC and MYB transcription factors. Plant Cell Physiol. 52(10), 1856-1871 (2011).

53. Abbruscato, P. et al. OsWRKY22, a monocot wrky gene, plays a role in the resistance response to blast. Mol. Plant Pathol. 13(8), 828-841 (2012).

54. Qiu, D. et al. OsWRKY13 mediates rice disease resistance by regulating defense-related genes in salicylate- and jasmonatedependent signaling. Mol. Plant-Microbe Interact. 20(5), 492-499 (2007).

55. Jing, S., Zhou, X., Song, Y. \& Yu, D. Heterologous expression of OsWRKY23 gene enhances pathogen defense and dark-induced leaf senescence in Arabidopsis. Plant Growth Regul. 58(2), 181-190 (2009).

56. Adamczyk, B. J. \& Fernandez, D. E. MIKC* MADS domain heterodimers are required for pollen maturation and tube growth in Arabidopsis. Plant Physiol. 149(4), 1713-1723 (2009).

57. Wen, K. et al. OsCPK21 is required for pollen late-stage development in rice. J. Plant Physiol. 240, 153000 (2019).

58. Phan, H. A., Li, S. F. \& Parish, R. W. MYB80, a regulator of tapetal and pollen development, is functionally conserved in crops. Plant Mol. Biol. 78(1-2), 171-183 (2012).

59. Pan, X. et al. OsMYB80 regulates anther development and pollen fertility by targeting multiple biological pathways. Plant Cell Physiol. 61(5), 988-1004 (2020).

60. Liu, X. Q. et al. The effect of the rice blast resistance gene Pi36 on the expression of disease resistance-related genes. Chin. Sci. Bull. 55(18), 1881-1888 (2010).

61. Mangelsen, E. et al. Phylogenetic and comparative gene expression analysis of barley (Hordeum vulgare) WRKY transcription factor family reveals putatively retained functions between monocots and dicots. BMC Genom. 9(1), 94 (2008).

62. Enfissi, E. M. A. et al. New plant breeding techniques and their regulatory implications: An opportunity to advance metabolomics approaches. J. Plant Physiol. 258-259, 153378 (2021).

63. Zhang, G. Target chromosome-segment substitution: A way to breeding design in rice. The Crop Journal 9(3), 658-668 (2021).

\section{Author contributions}

M.R.A.Z.; performed the experiment and drafted the manuscript, N.F.A.Z.; performed the experiment, N.G., S.H., N.M.A. and Z.A.M.H.; revised the draft manuscript, supervised and coordinated the experiments. All authors reviewed the manuscript.

\section{Competing interests}

The authors declare no competing interests.

Additional information

Supplementary Information The online version contains supplementary material available at https://doi.org/ 10.1038/s41598-021-99206-y.

Correspondence and requests for materials should be addressed to N.G.

Reprints and permissions information is available at www.nature.com/reprints.

Publisher's note Springer Nature remains neutral with regard to jurisdictional claims in published maps and institutional affiliations.

(c) Open Access This article is licensed under a Creative Commons Attribution 4.0 International cc. License, which permits use, sharing, adaptation, distribution and reproduction in any medium or format, as long as you give appropriate credit to the original author(s) and the source, provide a link to the Creative Commons licence, and indicate if changes were made. The images or other third party material in this article are included in the article's Creative Commons licence, unless indicated otherwise in a credit line to the material. If material is not included in the article's Creative Commons licence and your intended use is not permitted by statutory regulation or exceeds the permitted use, you will need to obtain permission directly from the copyright holder. To view a copy of this licence, visit http://creativecommons.org/licenses/by/4.0/.

(c) The Author(s) 2021 